\title{
ASYMPTOTIC EXPANSIONS ON MOMENTS OF THE FIRST LADDER HEIGHT IN MARKOV RANDOM WALKS WITH SMALL DRIFT
}

\author{
CHENG-DER FUH, ${ }^{*}$ National Central University and Academia Sinica
}

\begin{abstract}
Let $\left\{\left(X_{n}, S_{n}\right), n \geq 0\right\}$ be a Markov random walk in which $X_{n}$ takes values in a general state space and $S_{n}$ takes values on the real line $\mathbb{R}$. In this paper we present some results that are useful in the study of asymptotic approximations of boundary crossing problems for Markov random walks. The main results are asymptotic expansions on moments of the first ladder height in Markov random walks with small positive drift. In order to establish the asymptotic expansions we study a uniform Markov renewal theorem, which relates to the rate of convergence for the distribution of overshoot, and present an analysis of the covariance between the first passage time and the overshoot.
\end{abstract}

Keywords: Boundary crossing probability; ladder height distribution; Markov-dependent Wald martingale; overshoot; Poisson equation; uniform Markov renewal theory

2000 Mathematics Subject Classification: Primary 60K05

Secondary 60J10; 60K15

\section{Introduction}

Let $\left\{X_{n}, n \geq 0\right\}$ be a Markov chain on a general state space $\mathcal{X}$ with $\sigma$-algebra $\mathcal{A}$. Suppose that an additive component $S_{n}=\sum_{t=1}^{n} \xi_{t}$ with $S_{0}=0$, taking values on the real line $\mathbb{R}$, is adjoined to the chain such that $\left\{\left(X_{n}, S_{n}\right), n \geq 0\right\}$ is a Markov chain on $\mathcal{X} \times \mathbb{R}$ with

$$
\begin{aligned}
& \mathrm{P}\left\{\left(X_{n}, S_{n}\right) \in A \times(B+s) \mid\left(X_{n-1}, S_{n-1}\right)=(x, s)\right\} \\
& \quad=\mathrm{P}\left\{\left(X_{1}, S_{1}\right) \in A \times B \mid\left(X_{0}, S_{0}\right)=(x, 0)\right\}=\mathrm{P}(x, A \times B)
\end{aligned}
$$

for all $x \in \mathcal{X}, s \in \mathbb{R}, A \in \mathcal{A}$, and $B \in \mathcal{B}(\mathbb{R})$ (where $\mathcal{B}(\mathbb{R})$ is the Borel $\sigma$-algebra on $\mathbb{R}$ ). The chain $\left\{\left(X_{n}, S_{n}\right), n \geq 0\right\}$ is called a Markov random walk. For an initial distribution $v$ on $X_{0}$, let $\mathrm{P}_{v}$ denote the probability measure under the initial distribution $v$ on $X_{0}$ and let $\mathrm{E}_{v}$ denote the corresponding expectation. If $v$ is degenerate at $x$, we shall simply write $\mathrm{P}_{x}\left(\mathrm{E}_{x}\right)$ instead of $\mathrm{P}_{v}\left(\mathrm{E}_{v}\right)$. Some interesting examples related to the Markov models, (1.1), can be found in Kesten (1974), and Klüppelberg and Pergamenshchikov (2003).

Suppose that the Markov chain $\left\{X_{n}, n \geq 0\right\}$ has an invariant probability $\pi$. Let

$$
\tau=\tau_{+}=\inf \left\{n \geq 1: S_{n}>0\right\}
$$

be the first ascending ladder epoch of $S_{n}$, and denote the first positive value taken by the Markov random walk, $S_{\tau_{+}}$, as the first ladder height. For a given $a>0$, we want to establish asymptotic expansions, in terms of $\theta$ as $\theta \rightarrow 0$, of

$$
\mu_{\theta} \mathrm{E}_{\pi_{+}}^{\theta}\left(\tau_{+} S_{\tau_{+}}^{a}\right) \text { and } \mathrm{E}_{\pi_{+}}^{\theta} S_{\tau_{+}}^{a} .
$$

Received 13 September 2006; revision received 8 June 2007.

* Postal address: Institute of Statistical Science, Academia Sinica, Taipei 11529, Taiwan, ROC.

Email address: stcheng@stat.sinica.edu.tw 
Here $\mu_{\theta}$ denotes the mean value, with $\theta$ interpreted as the parameter representing various means of the Markov random walk $S_{1}$, and $\mathrm{E}_{\pi_{+}}^{\theta}$ denotes the expectation under invariant probability $\pi_{+}$of the ladder Markov chain in a family of distributions indexed by $\theta$, which will be defined precisely in Section 2. The main idea of obtaining the asymptotic expansions of (1.3) involves using the twist transformation for the Markov transition operator, the time-reversed Markov chain, and the ladder Markov random walk. To the author's knowledge, the essential step of characterizing constant terms in asymptotic expansions involving the solutions of the Poisson equations, to be defined in (2.8) and (2.9) precisely, is new.

The asymptotic expansions of (1.3) have important applications for deriving asymptotic approximations of boundary crossing problems for Markov random walks. In the case of simple random walks, Siegmund (1979), (1985, Chapter X) developed the so-called corrected diffusion approximations, by computing correction terms in the diffusion approximation, to approximate the first passage probabilities of $S_{n}$ and the expected values of the first ladder height in (1.3). That is, he considered the first ladder height in an exponential family of distributions, $\left\{F_{\theta}: \theta \in \Theta\right\}$, which may be written in the form $F_{\theta}(\mathrm{d} x)=\exp (\theta x-\Lambda(\theta)) F(\mathrm{~d} x)$, where $\Theta$ is the parameter space, $\Lambda(\theta)$ is the cumulant generating function, and $F$ denotes the distribution under $\theta=0$. Note that here we assume $\Theta$ contains a neighborhood of 0 . Let $\mathrm{P}^{\theta}(\mathrm{P})$ and $\mathrm{E}^{\theta}(\mathrm{E})$ denote the probability and the expectation, respectively, when the distribution of $X_{n}$ is $F_{\theta}(F)$. Under some regularity conditions, Siegmund (1979) showed that, for $a>0$,

$$
\mathrm{E}^{\theta} S_{\tau_{+}}^{a}=\mathrm{E} S_{\tau_{+}}^{a}+\frac{a}{a+1}\left(\mathrm{E} S_{\tau_{+}}^{a+1}\right) \theta+o(\theta)
$$

as $\theta \downarrow$ 0. By making use of the results in Siegmund (1982), (1988), Chang (1992) extended (1.4) to a high-order asymptotic expansion, with the $o(\theta)$ replaced by $C_{a} \theta^{2}+O\left(\theta^{3}\right)$, where $C_{a}$ is a constant depending on $a$, the distribution of overshoot, and the renewal function of the descending ladder random variables. Further refinements of (1.4) can be found in Lotov (1996), and Chang and Peres (1997) for Gaussian random walks, to which the coefficients are related to the celebrated Riemann zeta function. Using different techniques to those utilized by Chang (1992), Blanchet and Glynn (2006) proposed a method to compute the coefficients in the asymptotic expansion of the moments of the first ladder heights for non-Gaussian random walks (up to arbitrary order).

In the case of a finite state ergodic Markov chain, Asmussen (1989b) derived a first-order corrected diffusion approximation for one-barrier ruin problems in risk theory, while Fuh (1997) studied one-barrier and two-barrier boundary crossing probabilities, and derived a second-order corrected diffusion approximation in Markov random walks. To establish the approximations, they also derived first-order asymptotic expansions of (1.3). Glasserman and Kou (1995) studied the first passage times for rare sets in regenerative processes. For a general account of ruin probabilities, the reader is referred to Asmussen (2000) and the references therein.

An alternative approximation of the corrected diffusion approximation is the so-called large deviations approximation. In the case of Markov random walks, using the idea of large deviations and constructing a Markov chain extension of the classical Wald martingale family, Miller (1962a), (1962b) derived the asymptotic behavior of $\mathrm{P}\left\{\tau_{b}<\infty \mid X_{0}=x\right\}$ based on a Markov Wiener-Hopf factorization, where $\tau_{b}=\inf \left\{n \geq 1: S_{n}>b\right\}$ for $b>0$. This technique was further developed by Arndt (1980) to study asymptotic properties of the distribution of the supremum for a random walk on a Markov chain. Local limit theorems for the joint distribution $\mathrm{P}\left\{\tau_{b}=n, S_{\tau_{b}}-b \leq s, X_{\tau_{b}} \in \mathrm{d} y \mid X_{0}=x\right\}$ were derived by Lalley (1984). Hoglund (1991) combined these techniques with the idea of large deviations to study the ruin problems for finite 
state Markov random walks; while Chan and Lai (2003) provided saddle point approximations and nonlinear boundary crossing probabilities for general state Markov random walks.

Our motivation for providing asymptotic expansions of (1.3) stems from the approximation of boundary crossing probabilities in reflected Markov random walks. That is, let $W_{n}=$ $S_{n}-\min _{0 \leq k \leq n} S_{k}$ be the reflected Markov random walk with reflecting barrier at 0 . For $b>0$, define the stopping time $t=t_{b}=\inf \left\{n: W_{n}>b\right\}$. In a variety of contexts, for given $m \leq \infty$, we need to approximate the first passage probabilities, i.e.

$$
\mathrm{P}_{\pi}\{t \leq m\} \quad \text { and } \quad \mathrm{P}_{\pi}\left\{t \leq m \mid S_{m}=\zeta\right\}, \quad \zeta<b .
$$

It is known that, with some proper identifications, the first term of (1.5) is the probability that at least one among the first $m$ customers in a single-server Markov-modulated queue has a waiting time exceeding $b$; see Burman and Smith (1986) and Asmussen (1989a), (1989b), (2000, Chapter VI). The approximation of (1.5) is an essential step in the approximation of the distribution of the run length of a CUSUM test in autoregressive models and state space models; see Basseville and Nikiforov (1993, Chapter 7.3), and Fuh (2003).

As noted in (1.3) and (1.4), the theory developed here establishes an asymptotic expansion which can be expressed in terms of moments of Markov random walks. In order to characterize the coefficients in the expansion, we follow a similar idea to that used for the case of an independent and identically distributed (i.i.d.) increment. The idea of duality leads to that of time-reversed descending ladder Markov random walks and expected values of the ladder heights. The idea of exponential embedding leads to that of twist transformations of the transition probability operator. Owing to Markovian dependence, the constants also involve solutions of the Poisson equations; see Section 2 for details. However, in order to apply the results, we need to implement the coefficients in the expansion. In the case of i.i.d. increments, Chang (1992) derived an asymptotic expansion to which the constants depend on integrals of the whole renewal function. By making use of a technique in complex analysis, Blanchet and Glynn (2006) developed a method to compute the constants. In order to compute the constants developed in this paper (see Theorem 1 for details), we first need to extend Blanchet and Glynn's results to the Markovian case. Then, we need to develop a numerical method to compute solutions of the Poisson equations. In the setting of a MArP/PH/1 queue, computational tasks related to the ladder Markov random walks can be found in Asmussen (2003, Chapter XI). In general, the implementation issue is still an interesting open problem, and deserves further study. Moreover, after obtaining the coefficients in the expansion, we can gain insight into how the additional coefficient obtained in this paper improves upon a first-order expansion.

The remainder of the paper is organized as follows. In Section 2 we formulate the problem and state our main results: asymptotic expansions on moments of the first ladder height in Markov random walks with small positive drift. As an application of our main results, we also present an asymptotic approximation of the first term in (1.5). Motivated by the approximations of (1.3), we study a uniform Markov renewal theorem which relates to the rate of convergence for the distribution of overshoot in Section 3, and the rate of convergence for the covariance between first passage time and overshoot in Section 4. The proofs of our main results are given in Section 5.

\section{Asymptotic expansions on moments of the first ladder height}

Let $\left\{\left(X_{n}, S_{n}\right), n \geq 0\right\}$ be the Markov random walk on $\mathcal{X} \times \mathbb{R}$ as defined in (1.1), with transition probability kernel $\mathrm{P}(x, A \times B)$. The corresponding $m$-step transition kernel will be denoted by $\mathrm{P}^{m}$. For ease of notation, write $\mathrm{P}(x, A)=\mathrm{P}(x, A \times \mathbb{R})$ as the transition 
probability kernel of $\left\{X_{n}, n \geq 0\right\}$. For two transition probability kernels $Q(x, A)$ and $K(x, A)$, where $x \in \mathcal{X}, A \in \mathcal{A}$ and for all measurable functions $h(x), x \in \mathcal{X}$, define $Q h$ and $Q K$ by $Q h(x)=\int Q(x, \mathrm{~d} y) h(y)$ and $Q K(x, A)=\int K(x, \mathrm{~d} y) Q(y, A)$, respectively. Let $\mathcal{N}$ be the Banach space of measurable functions $h: \mathcal{X} \rightarrow \mathbb{C}$ with norm $\|h\|<\infty$. We also introduce the Banach space $\mathcal{B}$ of transition probability kernels $Q$ such that the operator norm $\|Q\|=\sup \{\|Q g\| ;\|g\| \leq 1\}$ is finite. Some prototypical norms considered in the literature are the supremum norm, the $L_{p}$ norm, the weighted variation norm, and the bounded Lipschitz norm, among others; the reader is referred to Kartashov (1996, pp. 4-6) and Fuh (2004) for further details. Define also the Césaro averages $P^{(n)}=\sum_{j=0}^{n} P^{j} / n$, where $P^{j}$ is a $j$-fold power of $P, P^{0}=P^{(0)}=I$ and $I$ is the identity operator on $\mathcal{B}$. A Markov chain $\left\{X_{n}, n \geq 0\right\}$ is said to be uniformly ergodic with respect to a given norm $\|\cdot\|$, if there exists a stochastic kernel $\Pi$ such that $P^{(n)} \rightarrow \Pi$ as $n \rightarrow \infty$ in the induced operator norm in $\mathscr{B}$.

As the theorems developed below are the same for each fixed norm, in the rest of the paper we will focus on a particular norm, namely, the weighted variation norm. To be more precise, let $w: \mathcal{X} \rightarrow[1, \infty)$ be a measurable function and define, for all measurable functions $h$, a weighted variation norm $\|h\|_{w}=\sup _{x \in \mathcal{X}}|h(x)| / w(x)$ and set $\mathcal{N}_{w}=\left\{h:\|h\|_{w}<\infty\right\}$. The corresponding norm in $\mathscr{B}_{w}$ is of the form $\|Q\|_{w}=\sup _{x \in \mathcal{X}} \int|Q|(x, \mathrm{~d} y) w(y) / w(x)$. The Markov chain $\left\{X_{n}, n \geq 0\right\}$ is called $w$-uniformly ergodic in the case of the weighted variation norm.

Recall $\tau_{+}$, defined in (1.2), and let $\tau_{+}^{n}=\inf \left\{k \geq \tau_{+}^{n-1}: S_{k}>S_{\tau_{+}^{n-1}}\right\}$ be the $n$th ascending ladder epoch of $S_{n}$. Let $\tau_{-}=\inf \left\{n \geq 1: S_{n} \leq 0\right\}$ be the first descending ladder epoch of $S_{n}$, and $\tau_{-}^{n}=\inf \left\{k \geq \tau_{-}^{n-1}: S_{k} \leq S_{\tau^{n-1}}\right\}$ be the $n$th descending ladder epoch of $S_{n}$, for $n=2,3, \ldots$ If $\mu>0, \tau_{+}^{n}$ is finite almost surely under the probability $\mathrm{P}\left\{X_{\tau_{+}} \in A \mid X_{0}=x\right\}$ and, therefore, the associated ladder heights $S_{\tau_{+}^{n}}$ are well-defined positive random variables. Furthermore, $\left\{\left(X_{\tau_{+}^{n}}, S_{\tau_{+}^{n}}\right), n \geq 0\right\}$ is a Markov chain, and it is the so-called ladder Markov random walk. When $\mu=0$, we can still define the ladder Markov chain using the property of uniform integrability in Theorem 5 of Fuh and Lai (1998). It is assumed throughout this paper that $\mathrm{P}_{x}\left(\tau_{+}<\infty\right)=1$ for all $x \in \mathcal{X}$. Let $\pi_{+}$denote the invariant measure, which is assumed to exist, of the transition probability kernel $\mathrm{P}_{+}(x, A \times \mathbb{R}):=\mathrm{P}\left\{X_{\tau_{+}} \in A, S_{\tau_{+}} \in \mathbb{R} \mid X_{0}=x\right\}$ of the ladder Markov chain $\left\{\left(X_{\tau_{+}^{n}}, S_{\tau_{+}^{n}}\right), n \geq 0\right\}$.

The following assumptions will be used throughout this paper.

Assumption 2.1. Assume that $\left\{X_{n}, n \geq 0\right\}$ is aperiodic, irreducible (with respect to a maximal irreducible measure $\varphi$ on $(\mathcal{X}, \mathcal{A}))$, and $w$-uniformly ergodic, i.e. there exists an invariant probability measure $\pi$ such that $\int w(y) \mathrm{d} \pi(y)<\infty$ and

$$
\begin{gathered}
\lim _{n \rightarrow \infty} \sup _{x}\left\{\frac{1}{w(x)}\left|\mathrm{E}_{x}\left(h\left(X_{n}\right)\right)-\int h(y) \mathrm{d} \pi(y)\right|: x \in \mathcal{X},|h| \leq w\right\}=0, \\
\sup _{x}\left\{\frac{\mathrm{E}_{x}\left(w\left(X_{1}\right)\right)}{w(x)}\right\}<\infty ;
\end{gathered}
$$

see Meyn and Tweedie (1993, Chapter 16). We also assume that $\left\{X_{\tau_{+}^{n}}, n \geq 0\right\}$ is aperiodic, irreducible, and w-uniformly ergodic with (2.1) and (2.2) satisfied.

Assumption 2.2. Assume that $\left\{X_{n}, n \geq 0\right\}$ satisfies the minorization condition (see Ney and Nummelin (1987)), i.e. there exists a $k \geq 1$, a probability measure $\Psi$ on $\mathcal{X} \times \mathbb{R}$, and a measurable function $h$ on $\mathcal{X}$ such that $\int h(x) \mathrm{d} \pi(x)>0, \Psi(\mathcal{X} \times \mathbb{R})=1, \int \Psi(\mathrm{d} x \times \mathbb{R}) h(x)>0$, and $\mathrm{P}^{k}(x, A \times B) \geq h(x) \Psi(A \times B)$, for all $x \in \mathcal{X}, A \in \mathcal{A}$, and $B \in \mathcal{B}(\mathbb{R})$. 
Assumption 2.3. Assume that $\mathrm{E}_{v}\left\{w\left(X_{1}\right)\left(1+\left|\xi_{1}\right|^{r}\right)\right\}<\infty$ for some sufficiently large $r$, where $v$ is an initial distribution of the Markov chain $\left\{X_{n}, n \geq 0\right\}$. Furthermore, we assume that there exists $\Theta \subset \mathbb{R}$ containing an interval of 0 such that, for all $\theta \in \Theta$,

$$
\sup _{x}\left\{\frac{\mathrm{E}_{x}\left(\exp \left(\theta \xi_{1}\right) w\left(X_{1}\right)\right)}{w(x)}\right\} \leq C<\infty
$$

for some $C>0$.

Assumption 2.4. Assume that $\mathrm{E}_{\pi} \xi_{1}=0, \sup _{x} \mathrm{E}_{x}\left|\xi_{1}\right|^{a+3}<\infty$, for some $a>0$, and that there exists an $\varepsilon>0$ such that $\inf _{x} \mathrm{P}_{\pi}\left\{\xi_{1} \leq-\varepsilon \mid X_{1}=x\right\}>0$.

Assumption 2.5. There exists a $\sigma$-finite measure $M$ on $(\mathcal{X}, \mathcal{A})$ such that, for all $x \in \mathcal{X}$, the probability measure $\mathrm{P}_{x}$ on $(\mathcal{X}, \mathcal{A})$ defined by $\mathrm{P}_{x}(A)=\mathrm{P}\left(X_{1} \in A \mid X_{0}=x\right)$ is absolutely continuous with respect to $M$, so that $\mathrm{P}_{x}(A)=\int_{A} p(x, y) \mathrm{d} M(y)$ for all $A \in \mathcal{A}$, where $p(x, \cdot)=\mathrm{dP}_{x} / \mathrm{d} M$.

Assumption 2.6. Assume that, for some $n_{0} \geq 1$,

$$
\int_{-\infty}^{\infty} \int_{x \in \mathcal{X}}\left|\mathrm{E}_{x}\left\{\exp \left(i \theta \xi_{1}\right)\right\}\right|^{n_{0}} \mathrm{~d} \pi(x) \mathrm{d} \theta<\infty .
$$

Remark 2.1. Assumption 2.1 implies that Assumption 2.2 holds; see Meyn and Tweedie (1993, Theorem 14.0.1). We include Assumption 2.2 here for the case of other norms. Under Assumptions 2.1 and 2.2, and using the fact that $\varphi$ is $\sigma$-finite, Theorem 1.1 of Kartashov (1996) shows that $\mathrm{P}$ has a unique stationary projector $\Pi$ in the sense that $\Pi^{2}=\Pi=P \Pi=\Pi \mathrm{P}$, and $\Pi(x, A)=\pi(A)$ for all $x \in \mathcal{X}$ and $A \in \mathcal{A}$. Under irreducibility and aperiodicity, (2.1) implies that there exists a $C>0$ and $0<\rho<1$ such that, for all $h \in \mathbb{B}$, the Banach space of measurable functions, and $n \geq 1$,

$$
\sup _{x}\left\{\frac{1}{w(x)}\left|\mathrm{E}_{x}\left(h\left(X_{n}\right)\right)-\int h(y) \mathrm{d} \pi(y)\right|\right\} \leq C \rho^{n}\|h\|_{w} ;
$$

see Meyn and Tweedie (1993, pp. 382-383).

Remark 2.2. Assumption 2.3 is a moment condition in the sense of the weighted variation norm of $\xi_{1}$. Assumption 2.4 implies that $\mathrm{E}_{\pi} S_{\tau_{+}}^{a+2}<\infty$ for $a>0$; see Fuh and Lai (1998, Theorem 5). The existence of the transition probability density in Assumption 2.5 will be used to construct the time-reversed Markov chains. Note that Assumption 2.5 holds in most applications.

Remark 2.3. Assumption 2.6 implies that, for all $n \geq n_{0}, S_{n}$ has a bounded probability density function for given $X_{n}$. Instead of assuming Assumption 2.6, we may assume the following extension of Cramér's (strong nonlattice) condition: $g(\theta):=\inf _{|v|>\theta}\left|1-\mathrm{E}_{\pi}\left\{\exp \left(\mathrm{i} v S_{1}\right)\right\}\right|>0$ for all $\theta>0$. We also assume that the conditional Cramér's (strong nonlattice) condition is satisfied, which states that there exists an $m \geq 1$ such that

$$
\limsup _{|\theta| \rightarrow \infty}\left|\mathrm{E}\left\{\exp \left(i \theta S_{m}\right) \mid X_{0}, X_{m}\right\}\right|<1 .
$$

Here, we assume that Assumption 2.6 holds for simplicity. Some practical examples which satisfy Assumptions 2.1-2.6 can be found in Fuh (2003, Section 6). 
Remark 2.4. It is known (see Alsmeyer (2000)) that the ladder Markov random walk, $\left\{\left(X_{\tau_{+}^{n}}, S_{\tau_{+}^{n}}\right), n \geq 0\right\}$, satisfies Assumption 2.2. The uniformly strong nonlattice for the ladder random walk and the exponential moment condition, Assumption 2.3, for the ladder random walk can be found in Lemma 13 and Lemma 14 of Fuh (2004), respectively. In Section 2 of Fuh and Lai (2001) and Section 3 of Fuh (2004), it was shown, in some interesting examples, how the $w$-uniform ergodicity of the ladder Markov chain can be established. In general, the $w$-uniform ergodicity of the ladder Markov chain is an open problem.

For $b>0$, define the first passage time of the Markov random walk $S_{n}$ as

$$
\tau_{b}=\tau(b)=\inf \left\{n: S_{n}>b\right\} \quad\left(\tau_{+}=\tau(0)\right),
$$

and the residual at $b$ is defined as

$$
R_{b}=R(b)=S_{\tau_{b}}-b
$$

Under Assumptions 2.1-2.6, we can apply Corollary 2 of Alsmeyer (1997) to show that, as $b \rightarrow \infty,\left(X_{\tau_{b}}, S_{\tau_{b}}-b\right)$ has the limiting distribution $\left(X_{\infty}, R_{\infty}\right)$, which is defined by

$$
\mathrm{P}\left\{X_{\infty} \in A, R_{\infty}>s\right\}=\frac{1}{\mathrm{E}_{\pi_{+}} S_{\tau_{+}}} \int_{s}^{\infty} \mathrm{P}_{\pi_{+}}\left\{X_{\tau_{+}} \in A, S_{\tau_{+}}>u\right\} \mathrm{d} u
$$

for every $A \in \mathcal{A}$ and $s>0$.

To present our main results, we need to consider time-reversed descending ladder Markov random walks, study solutions of the Poisson equations, and define the twist transformation of the transition probability operator. These are given in the following three paragraphs, respectively.

By Assumption 2.5, the invariant probability measure $\pi$ of the Markov chain $\left\{X_{n}, n \geq 0\right\}$ has a positive density function with respect to $M$. Without any confusion, we still denote it as $\pi$ here and in the sequel. As in Section 4 of Fuh and Lai (1998), we consider the time-reversed (dual) process $\left\{\left(\tilde{X}_{n}, \tilde{S}_{n}\right), n \geq 0\right\}$ of $\left\{\left(X_{n}, S_{n}\right), n \geq 0\right\}$ with transition kernel

$$
\tilde{\mathrm{P}}(y, \mathrm{~d} x \times \mathrm{d} s)=\frac{\pi(x)}{\pi(y)} \mathrm{P}(x, \mathrm{~d} y \times \mathrm{d} s) .
$$

Note that $\left\{\tilde{X}_{n}, n \geq 0\right\}$ and $\left\{X_{n}, n \geq 0\right\}$ have the same invariant probability measure $\pi$. Let $\tilde{\tau}_{-}^{0}=0$ and $\tilde{\tau}_{-}=\inf \left\{n \geq 1: \tilde{S}_{n} \leq 0\right\}$, and, for $n>1$, define the $n$th weakly descending ladder epoch as $\tilde{\tau}_{-}^{n}=\inf \left\{k \geq \tilde{\tau}_{-}^{n-1}: \tilde{S}_{k} \leq \tilde{S}_{\tilde{\tau}^{n-1}}\right\}$. The same assumptions will be made for $\left\{\left(\tilde{X}_{\tilde{\tau}_{-}^{n}}, \tilde{S}_{\tilde{\tau}_{-}^{n}}\right), n \geq 0\right\}$ as made for $\left\{\left(X_{\tau_{+}^{n}}, S_{\tau_{+}^{n}}\right), \bar{n} \geq 0\right\}$. For $x \in \mathcal{X}$, define the renewal measure by

$$
\tilde{U}_{x,-}(A, B):=\sum_{n=0}^{\infty} \tilde{\mathrm{P}}_{x}\left\{\tilde{\tau}_{-}^{n}<\infty, \tilde{X}_{\tilde{\tau}_{-}^{n}} \in A, \tilde{S}_{\tilde{\tau}_{-}^{n}} \in B\right\}
$$

for all $A \in \mathcal{A}$ and Borel subsets $B \subset[0, \infty)$, and let

$$
\tilde{U}_{x,-}(A, v):=\sum_{n=0}^{\infty} \tilde{\mathrm{P}}_{x}\left\{\tilde{\tau}_{-}^{n}<\infty, \tilde{X}_{\tilde{\tau}_{-}^{n}} \in A,-\tilde{S}_{\tilde{\tau}_{-}^{n}} \leq v\right\}
$$

be the renewal function corresponding to the renewal process $\left\{-\tilde{S}_{\tilde{\tau}_{-}^{n}}, n=0,1, \ldots\right\}$. We simply denote $\tilde{U}_{x,-}(A, v)$ as $\tilde{U}_{x,-}(v)$ if $A=\mathcal{X}$ in (2.6). For a given $a>0$, define

$$
\alpha_{x}^{a}=\int_{[0, \infty)}\left(\mathrm{E}_{x} R_{b}^{a}-\mathrm{E}_{\pi_{+}} R_{\infty}^{a}\right) \tilde{U}_{x,-}(\mathrm{d} b) \quad \text { and } \quad \alpha^{a}=\int_{x \in \mathcal{X}} \alpha_{x}^{a} \mathrm{~d} \pi(x) .
$$


Let $g_{1}: \mathcal{X} \rightarrow \mathbb{R}$ be a solution of the Poisson equation

$$
g_{1}(x)-\mathrm{E}_{x} g_{1}\left(X_{\tau_{+}}\right)=\mathrm{E}_{x} S_{\tau_{+}}-\mathrm{E}_{\pi_{+}} S_{\tau_{+}}
$$

for almost every (with respect to $M) x \in \mathcal{X}$, with $\mathrm{E}_{\pi_{+}} g_{1}\left(X_{\tau_{+}}\right)=0$, and let $g_{2}$ be a solution of the Poisson equation

$$
\begin{aligned}
g_{2}(x)-\mathrm{E}_{x} g_{2}\left(X_{\tau_{+}}\right)= & \mathrm{E}_{x}\left(S_{\tau_{+}}-\mathrm{E}_{\pi_{+}} S_{\tau_{+}}+g_{1}\left(X_{\tau_{+}}\right)-g_{1}(x)\right)^{2} \\
& -\mathrm{E}_{\pi_{+}}\left(S_{\tau_{+}}-\mathrm{E}_{\pi_{+}} S_{\tau_{+}}+g_{1}\left(X_{\tau_{+}}\right)-g_{1}\left(X_{0}\right)\right)^{2}
\end{aligned}
$$

for almost every $x$, with $\mathrm{E}_{\pi_{+}} g_{2}\left(X_{\tau_{+}}\right)=0$. Note that under Assumptions 2.1-2.4, the solutions of (2.8) and (2.9) exist via (2.2) and (2.3) of Fuh and Zhang (2000), and Theorem 17.4.2 of Meyn and Tweedie (1993). Now, define

$$
\begin{aligned}
& \alpha_{1}\left(X_{\tau_{+}}\right)=g_{1}\left(X_{\tau_{+}}\right)-g_{1}\left(X_{0}\right), \\
& \alpha_{2}\left(X_{\tau_{+}}\right)=g_{2}\left(X_{\tau_{+}}\right)-g_{2}\left(X_{0}\right) .
\end{aligned}
$$

For $z \in \mathbb{C}$, define linear operators $\boldsymbol{P}_{z}, \boldsymbol{P}, v_{*}$, and $\boldsymbol{Q}$ on $\mathcal{N}$ by

$$
\begin{aligned}
\left(\boldsymbol{P}_{z} h\right)(x) & =\mathrm{E}\left[h\left(X_{1}\right) \mathrm{e}^{z \xi_{1}} \mid X_{0}=x\right], & (\boldsymbol{P} h)(x) & =\mathrm{E}\left[h\left(X_{1}\right) \mid X_{0}=x\right], \\
\mathcal{v}_{*} h & =\mathrm{E}_{v}\left\{h\left(X_{0}\right)\right\}, & \boldsymbol{Q} h & =\int h(y) \mathrm{d} \pi(y) .
\end{aligned}
$$

Proposition 1 of Fuh (2004) shows that there exists sufficiently small $\delta>0$, such that, for $|z| \leq \delta, \mathcal{N}=\mathcal{N}_{1}(z) \oplus \mathcal{N}_{2}(z)$ and

$$
\boldsymbol{P}_{z} \boldsymbol{Q}_{z} h=\lambda(z) \boldsymbol{Q}_{z} h \quad \text { for all } h \in \mathcal{N},
$$

where $\mathcal{N}_{1}(z)$ is a one-dimensional subspace of $\mathcal{N}, \lambda(z)$ is the eigenvalue of $\boldsymbol{P}_{z}$ with corresponding eigenspace $\mathcal{N}_{1}(z)$, and $\boldsymbol{Q}_{z}$ is the parallel projection of $\mathcal{N}$ onto the subspace $\mathcal{N}_{1}(z)$ in the direction of $\mathcal{N}_{2}(z)$. For the structure of $\mathcal{N}_{1}(z)$ and $\mathcal{N}_{2}(z)$, and other results of the perturbation theory for Markovian operators, the reader is referred to Appendix A of Fuh and Lai (2001) and Proposition 1 of Fuh (2004) for details.

Let $h_{1} \in \mathcal{N}$ be the constant function $h_{1} \equiv 1$ and let $r(x ; z)=\left(\boldsymbol{Q}_{z} h_{1}\right)(x)$. From (2.12), it follows that $r(\cdot ; z)$ is an eigenfunction of $\boldsymbol{P}_{z}$ associated with the eigenvalue $\lambda(z)$, i.e. $r(\cdot ; z)$ generates the one-dimensional eigenspace $\mathcal{N}_{1}(z)$. In particular, when $z=\theta \in \mathbb{R}$ such that there exists a $\delta>0$ and $|\theta| \leq \delta$, define the 'twisting' transformation by

$$
\mathrm{P}^{\theta}(x, \mathrm{~d} y \times \mathrm{d} s)=\frac{r(y ; \theta)}{r(x ; \theta)} \mathrm{e}^{-\Lambda(\theta)+\theta s} \mathrm{P}(x, \mathrm{~d} y \times \mathrm{d} s), \quad \text { where } \Lambda=\log \lambda .
$$

Then $\mathrm{P}^{\theta}$ is the transition probability of a Markov random walk $\left\{\left(X_{n}^{\theta}, S_{n}^{\theta}\right), n \geq 0\right\}$, with invariant probability $\pi^{\theta}$. Let $\mathrm{E}_{v}^{\theta}$ be the expectation under $\mathrm{P}_{v}^{\theta}$. The function $\Lambda(\theta)$ is normalized so that $\Lambda(0)=\dot{\Lambda}(0)=0$, where $\dot{\Lambda}$ denotes the first derivative of $\Lambda$ with respect to $\theta$. Then $\mathrm{P}^{0}=\mathrm{P}$ is the transition probability of the Markov random walk $\left\{\left(X_{n}, S_{n}\right), n \geq 0\right\}$ with invariant probability $\pi$. Here, and in the sequel, we denote by $\mathrm{P}_{v}^{\theta}$ the probability measure of the Markov random walk $\left\{\left(X_{n}^{\theta}, S_{n}^{\theta}\right), n \geq 0\right\}$, with transition probability kernel (2.13) and initial distribution $\nu^{\theta}$. For simplicity of notation, we denote $\nu^{\theta}:=v$ and $\pi^{\theta}:=\pi$, and delete $\theta$ in $\left\{\left(X_{n}^{\theta}, S_{n}^{\theta}\right), n \geq 0\right\}$ if it is under $\mathrm{P}^{\theta}$ or $\mathrm{E}^{\theta}$. Since $r(x ; 0)=1$, the continuity property 
of $r(x ; \theta)$ implies that there exists a $\delta>0$ and, thus, for $|\theta| \leq \delta$, we have $r(x ; \theta)>0$ (or $1 / r(x ; \theta)<\infty)$ uniformly for all $x \in \mathcal{X}$. For given $\theta$, let $\left\{\left(X_{\tau_{+}^{n}}^{\theta}, S_{\tau_{+}^{n}}^{\theta}\right), n \geq 0\right\}$ be the ascending ladder Markov random walk and set the invariant measure $\pi_{+}^{\theta}=\pi_{+}$for simplicity. Note that $\pi_{+}$has a probability density with respect to $M$ which, abusing the notation a little, we will denote by $\pi_{+}$again.

By Proposition 1 of Fuh (2004), it is known that $\Lambda$ is a strictly convex and real analytic function for which $\dot{\Lambda}(\theta)=\mathrm{E}_{\pi}^{\theta} \xi_{1}^{\theta}$. Therefore, $\mathrm{E}_{\pi}^{\theta} \xi_{1}^{\theta}$ is less than, equal to, or greater than 0 if and only if $\theta$ is less than, equal to, or greater than 0 . For any $\theta \neq 0$ and $|\theta|<\delta$, there is at most one value of $\theta^{\prime}$ with $\left|\theta^{\prime}\right|<\delta$, necessarily of opposite sign, for which $\Lambda(\theta)=\Lambda\left(\theta^{\prime}\right)$. Assume that such a $\theta^{\prime}$ exists, we may let $\theta_{0}=\min \left(\theta, \theta^{\prime}\right)$ and $\theta_{1}=\max \left(\theta, \theta^{\prime}\right)$ such that $\theta_{0}<0<\theta_{1}$ and $\Lambda\left(\theta_{0}\right)=\Lambda\left(\theta_{1}\right)$. Denote $\mu_{\theta}=\dot{\Lambda}(\theta)$, and let $\Delta=\theta_{1}-\theta_{0}$. We also assume, without loss of generality, that $\sigma^{2}=\ddot{\Lambda}(0)=1$, where $\ddot{\Lambda}$ denotes the second derivative of $\Lambda$ with respect to $\theta$.

Theorem 2.1. Let $\left\{\left(X_{n}, S_{n}\right), n \geq 0\right\}$ be a Markov random walk satisfying Assumptions 2.1-2.6 and denote by $\left\{\left(X_{n}^{\theta}, S_{n}^{\theta}\right), n \geq 0\right\}$ the Markov random walk induced by (2.13) with $\mathrm{E}_{\pi}^{\theta} S_{1}>0$. Then, for any $a>0$, as $\theta \downarrow 0$, we have

$$
\begin{aligned}
\mu_{\theta} \mathrm{E}_{\pi_{+}}^{\theta}\left(\tau_{+} S_{\tau_{+}}^{a}\right)= & \frac{1}{a+1} \mathrm{E}_{\pi_{+}} S_{\tau_{+}}^{a+1} \\
& +\left(\frac{1}{a+2} \mathrm{E}_{\pi_{+}} S_{\tau_{+}}^{a+2}+\mathrm{E}_{\pi_{+}}\left(S_{\tau_{+}}^{a+1} \alpha_{1}\left(X_{\tau_{+}}\right)\right)+\alpha^{a}\right) \theta+O\left(\theta^{2}\right) .
\end{aligned}
$$

Hence, as $\theta \downarrow 0$,

$$
\begin{aligned}
\mathrm{E}_{\pi_{+}}^{\theta} S_{\tau_{+}}^{a}= & \mathrm{E}_{\pi_{+}} S_{\tau_{+}}^{a}+\left(\frac{a}{a+1} \mathrm{E}_{\pi_{+}} S_{\tau_{+}}^{a+1}+\mathrm{E}_{\pi_{+}}\left(S_{\tau_{+}}^{a} \alpha_{1}\left(X_{\tau_{+}}\right)\right)\right) \theta \\
& +\frac{1}{2}\left(\frac{a}{a+2} \mathrm{E}_{\pi_{+}} S_{\tau_{+}}^{a+2}+\mathrm{E}_{\pi_{+}}\left(S_{\tau_{+}}^{a+1} \alpha_{1}\left(X_{\tau_{+}}\right)+S_{\tau_{+}}^{a} \alpha_{2}\left(X_{\tau_{+}}\right)\right)-\alpha^{a}\right) \theta^{2}+O\left(\theta^{3}\right)
\end{aligned}
$$

where $\alpha_{1}\left(X_{\tau_{+}}\right)$and $\alpha_{2}\left(X_{\tau_{+}}\right)$are defined in (2.10) and (2.11), respectively.

The idea of the proof involves the twist transformation for the Markov transition operator in (2.13), the time-reversed Markov chains from (2.4)-(2.9), and Taylor's expansion with respect to $\theta$. The details will be given in Section 5 .

Remark. To compare the asymptotic expansion (2.15) to (4.3) of Chang (1992), we observe that there is an extra term $\mathrm{E}_{\pi_{+}}\left(S_{\tau_{+}}^{a} \alpha_{1}\left(X_{\tau_{+}}\right)\right)$, the joint moment of the first ladder height and the solution of the Poisson equation (2.8), in the first-order approximation; and an extra term $\mathrm{E}_{\pi_{+}}\left(S_{\tau_{+}}^{a+1} \alpha_{1}\left(X_{\tau_{+}}\right)+S_{\tau_{+}}^{a} \alpha_{2}\left(X_{\tau_{+}}\right)\right)$, the joint moment of the first ladder height and the solutions of the Poisson equations (2.8) and (2.9), in the second-order approximation. An interpretation for these extra terms can be described as follows: note that

$$
\frac{a}{a+1} \mathrm{E}_{\pi_{+}} S_{\tau_{+}}^{a+1}+\mathrm{E}_{\pi_{+}}\left(S_{\tau_{+}}^{a} \alpha_{1}\left(X_{\tau_{+}}\right)\right)=-\frac{1}{a+1} \mathrm{E}_{\pi_{+}} S_{\tau_{+}}^{a+1}+\mathrm{E}_{\pi_{+}}\left(S_{\tau_{+}}^{a}\left(S_{\tau_{+}}+\alpha_{1}\left(X_{\tau_{+}}\right)\right)\right)
$$

and $S_{n}+\alpha_{1}\left(X_{n}\right)$ is a martingale since $\mu=0$; see Meyn and Tweedie (1993, Theorem 17.4.3). Using the fact that $\alpha_{1}\left(X_{n}\right)=0$ in the case of simple random walks, we may regard the extra constant $\alpha_{1}\left(X_{\tau_{+}}\right)$as being due to Markovian dependence, which reflects the martingale structure 
of Markov random walks. The same interpretation can also be applied to $S_{n}^{2}+\alpha_{2}\left(X_{n}\right)$, which forms a quadratic martingale; see Fuh and Zhang (2000, Theorem 3). Note that the constant $\alpha^{a}$ depends on the distribution of overshoot and the renewal function for the time-reversed descending ladder Markov chains.

Using the tilting formula, (2.13), and Theorem 2.1, we give an asymptotic approximation of the first term in (1.5) as follows. Under the conditions of Theorem 2.1, assume that $\theta_{0} \uparrow 0$, $b \rightarrow \infty$, and $m \rightarrow \infty$ in such a way that, for some $\delta>0$ and some $k$, we have

$$
\left|\theta_{0}\right|^{1+\delta} b \rightarrow \infty, \quad\left|\theta_{0}\right|^{k} m \rightarrow 0, \quad \text { and } \quad m \geq \frac{b}{\mu_{1}}(1+\delta) .
$$

Then

$$
\begin{aligned}
\mathrm{P}_{\pi}^{\theta_{0}}\left\{t_{b} \leq m\right\} \cong \exp \left[-\Delta\left(b+\rho_{+}-\rho_{-}\right)\right] & \left(\Delta\left|\mu_{0}\right|\left(m-\frac{b+\left(\rho_{+}+\zeta_{+}\right)-\left(\rho_{-}+\zeta_{-}\right)}{\mu_{1}}\right)\right. \\
& \left.+3\left(1+\Delta \mathrm{E}_{\pi_{+}} \alpha_{1}\left(X_{\tau_{+}}\right)\right)-\frac{2}{3} \gamma \Delta\right),
\end{aligned}
$$

where $\mu_{0}=\dot{\Lambda}\left(\theta_{0}\right), \mu_{1}=\dot{\Lambda}\left(\theta_{1}\right), \gamma=\mathrm{E}_{\pi} \xi_{1}^{3}$, and

$$
\begin{aligned}
\rho_{+} & =\frac{\mathrm{E}_{\pi_{+}} S_{\tau_{+}}^{2}}{2 \mathrm{E}_{\pi_{+}} S_{\tau_{+}}}, & \rho_{-} & =\frac{\tilde{\mathrm{E}}_{\pi_{-}} \tilde{S}_{\tilde{\tau}_{-}}^{2}}{2 \tilde{\mathrm{E}}_{\pi_{-}} \tilde{S}_{\tilde{\tau}_{-}}}, \\
\zeta_{+} & =\frac{\mathrm{E}_{\pi_{+}}\left(S_{\tau_{+}}^{2} \alpha_{1}\left(X_{\tau_{+}}\right)\right)}{\mathrm{E}_{\pi_{+}} S_{\tau_{+}}}, & \zeta_{-} & =\frac{\tilde{\mathrm{E}}_{\pi_{-}}\left(\tilde{S}_{\tilde{\tau}_{-}}^{2} \tilde{\alpha}_{1}\left(\tilde{X}_{\tilde{\tau}_{-}}\right)\right)}{\tilde{\mathrm{E}}_{\pi_{-}} \tilde{S}_{\tilde{\tau}_{-}}},
\end{aligned}
$$

with $\tilde{\alpha}_{1}\left(\tilde{X}_{\tilde{\tau}_{-}}\right)$defined as $\alpha_{1}\left(X_{\tau_{+}}\right)$in (2.10) for the time-reversed ladder Markov random walk $\left\{\left(\tilde{X}_{\tilde{\tau}_{-}}, \tilde{S}_{\tilde{\tau}_{-}}\right), n \geq 0\right\}$.

Note that approximation (2.16) generalizes Theorem 3 of Siegmund (1988) to the case of reflected Markov random walks. A formal proof of (2.16) along with high-order asymptotic approximations of (1.5), and its applications to the CUSUM change point detection procedure will be published in a forthcoming paper.

\section{Rate of convergence for the distribution of overshoot}

Using the same notation as in Section 2, we define the renewal measure for Markov random walks as

$$
U_{\nu}(A, B)=\sum_{n=0}^{\infty} \mathrm{P}_{\nu}\left\{X_{n} \in A, S_{n} \in B\right\},
$$

where $A \in \mathcal{A}$ and $B \in \mathcal{B}$. When $v$ is degenerated at $x$, we simply denote it as $U_{x}(A, \cdot)$. Then under Assumption 2.5, for fixed $B \in \mathcal{B}, U_{x}(A, B)$ is absolutely continuous with respect to $M$, with density function $u(y ; x, B)$ so that $U_{x}(A, B)=\int_{A} u(y ; x, B) \mathrm{d} M(y)$. It is simple to note that the measure $\mathrm{P}_{+}(x, \cdot \times \mathbb{R})$ on $(\mathcal{X}, \mathcal{A})$ is also absolutely continuous with respect to $M$ for every $x \in \mathcal{X}$. Likewise, the renewal measure $U_{x,+}$, as defined in (3.1), associated with the ascending ladder random walk initialized at $(x, 0)$ also has density function $u_{+}(\cdot ; x, B)$ with respect to $M$, i.e. $U_{x,+}(A, B)=\int_{A} u_{+}(y ; x, B) \mathrm{d} M(y)$. When $B=(-\infty, t]$, we simply denote it as $u_{+}(y ; x, t)$. Assumption 2.5 also implies that, for every $x \in \mathcal{X}$, there exists a $p_{-}(x ; \cdot)$ such that $\mathrm{P}\left(X_{\tau_{-}} \in A \mid X_{0}=x\right)=\int_{A} p_{-}(x ; y) \mathrm{d} M(y)$ for all $A \in \mathcal{A}$. For given $B \in \mathscr{B}((-\infty, 0))$, 
define $G_{-}(x, y ; B)=p_{-}(x, y) \mathrm{P}\left\{S_{\tau_{-}} \in B \mid X_{0}=x, X_{\tau_{-}}=y\right\}$. Consider the time-reversed (dual) process $\left\{\left(\tilde{X}_{n}, \tilde{S}_{n}\right), n \geq 0\right\}$ and define $\tilde{p}_{-}$and $\tilde{G}_{-}$for the dual process in the same way as $p_{-}$and $G_{-}$are defined for $\left\{\left(X_{n}, S_{n}\right), n \geq 0\right\}$. Let $\widehat{G}_{-}(x, y ; B)=\tilde{G}_{-}(y, x ; B) \pi(y) / \pi(x)$, $\widehat{U}_{-}=\sum_{n=0}^{\infty} \widehat{G}_{-}^{* n}$, where $*$ denotes the convolution of two transition kernels $F_{1}(x, y ; \cdot)$ and $F_{2}(x, y ; \cdot)$, with $\widehat{G}_{-}^{* 1}=\widehat{G}$ and $\widehat{G}_{-}^{* 0}$ being the kernel that puts all its mass at 0 .

By making use of the exponential rate of convergence for the Markov renewal theory in Fuh (2004), the next result states the exponential rate of convergence for the distribution of overshoot.

Theorem 3.1. Let $\left\{\left(X_{n}, S_{n}\right), n \geq 0\right\}$ be a Markov random walk satisfying Assumptions 2.1-2.6 and denote by $\left\{\left(X_{n}^{\theta}, S_{n}^{\theta}\right), n \geq 0\right\}$ the Markov random walk induced by (2.13) with $\mathrm{E}_{\pi}^{\theta} S_{1}>0$. Then there exists an $r>0, \theta^{*}>0$, and $C$ such that, for any $x \in \mathcal{X}$ and $A \in \mathcal{A}$,

$$
\left|\mathrm{P}_{x}^{\theta}\left\{X_{\tau_{b}} \in A, R_{b} \leq s\right\}-\mathrm{P}^{\theta}\left\{X_{\infty} \in A, R_{\infty} \leq s\right\}\right| \leq C \mathrm{e}^{-r(s+b)}
$$

for all $s, b \geq 0$ and $\theta \in\left[0, \theta^{*}\right]$.

Proof. For given $\theta \in\left(0, \theta^{*}\right], x, y \in \mathcal{X}$, and $B \in \mathcal{B}((-\infty, 0))$, define $u_{+}^{\theta}(y ; x, B)$ as that in the first paragraph of this section for the Markov random walk $\left\{\left(X_{\tau_{n}}^{\theta}, S_{\tau_{n}}^{\theta}\right), n \geq 0\right\}$. Since, by (3.15) of Fuh and Lai (2001),

$$
\mathrm{P}_{x}^{\theta}\left\{X_{\tau_{b}} \in A, S_{\tau_{b}}-b>s\right\}=\int_{X} \int_{[0, b)} \mathrm{P}_{y}^{\theta}\left\{X_{\tau_{+}} \in A, S_{\tau_{+}}>b+s-t\right\} u_{+}^{\theta}(y ; x, \mathrm{~d} t) \mathrm{d} M(y)
$$

for any $x \in \mathcal{X}$ and $A \in \mathcal{A}$, and since, by (2.3),

$$
\begin{aligned}
\mathrm{P}^{\theta}\left\{X_{\infty} \in A, R_{\infty}>s\right\} & =\int_{s}^{\infty} \mathrm{P}_{\pi_{+}}^{\theta}\left\{X_{\tau_{+}} \in A, S_{\tau_{+}}>t\right\} \frac{\mathrm{d} t}{\mathrm{E}_{\pi_{+}}^{\theta} S_{\tau_{+}}} \\
& =\int_{x} \int_{-\infty}^{b} \mathrm{P}_{y}^{\theta}\left\{X_{\tau_{+}} \in A, S_{\tau_{+}}>b+s-t\right\} \frac{\pi_{+}(y) \mathrm{d} t}{\mathrm{E}_{\pi_{+}}^{\theta} S_{\tau_{+}}} \mathrm{d} M(y),
\end{aligned}
$$

we have, for any $x \in \mathcal{X}$ and $A \in \mathcal{A}$,

$$
\begin{aligned}
\mathrm{P}_{x}^{\theta}\left\{X_{\tau_{b}} \in A, S_{\tau_{b}}-b>s\right\}-\mathrm{P}^{\theta}\left\{X_{\infty} \in A, R_{\infty}>s\right\} \\
=\int_{x} \int_{[0, b)} \mathrm{P}_{y}^{\theta}\left\{X_{\tau_{+}} \in A, S_{\tau_{+}}>b+s-t\right\}\left(u_{+}^{\theta}(y ; x, \mathrm{~d} t)-\frac{\pi_{+}(y) \mathrm{d} t}{\mathrm{E}_{\pi_{+}}^{\theta} S_{\tau_{+}}}\right) \mathrm{d} M(y) \\
\quad-\int_{x} \int_{-\infty}^{0} \mathrm{P}_{y}^{\theta}\left\{X_{\tau_{+}} \in A, S_{\tau_{+}}>b+s-t\right\} \frac{\pi_{+}(y) \mathrm{d} t}{\mathrm{E}_{\pi_{+}}^{\theta} S_{\tau_{+}}} \mathrm{d} M(y) \\
\quad:=J_{1}-J_{2} .
\end{aligned}
$$

For all $x, y \in \mathcal{X}$, define

$$
\eta_{\theta}(y ; x, b)=u_{+}^{\theta}(y ; x, b)-\frac{\pi_{+}(y) b}{\mathrm{E}_{\pi_{+}}^{\theta} S_{\tau_{+}}}-\frac{\pi_{+}(y) \mathrm{E}_{\pi_{+}}^{\theta} S_{\tau_{+}}^{2}}{2\left(\mathrm{E}_{\pi_{+}}^{\theta} S_{\tau_{+}}\right)^{2}} .
$$

Then

$$
J_{1}=\int_{X} \int_{[0, b)} \mathrm{P}_{y}^{\theta}\left\{X_{\tau_{+}} \in A, S_{\tau_{+}}>b+s-t\right\} \eta_{\theta}(y ; x, \mathrm{~d} t) \mathrm{d} M(y),
$$


and integration by parts gives

$$
\begin{aligned}
J_{1}= & \int_{X}\left(\eta_{\theta}(y ; x, b) \mathrm{P}_{y}^{\theta}\left\{X_{\tau_{+}} \in A, S_{\tau_{+}}>s\right\}\right. \\
& \left.\quad+\frac{\pi_{+}(y) \mathrm{E}_{\pi_{+}}^{\theta} S_{\tau_{+}}^{2}}{2\left(\mathrm{E}_{\pi_{+}}^{\theta} S_{\tau_{+}}\right)^{2}} \mathrm{P}_{y}^{\theta}\left\{X_{\tau_{+}} \in A, S_{\tau_{+}}>b+s\right\}\right) \mathrm{d} M(y) \\
& -\int_{x} \int_{[0, b)} \mathrm{P}_{y}^{\theta}\left\{X_{\tau_{+}} \in A, S_{\tau_{+}} \in b+s-\mathrm{d} t\right\} \eta_{\theta}(y ; x, t) \mathrm{d} M(y) .
\end{aligned}
$$

To find an upper bound of $\left|J_{1}\right|$, note that Theorem 1 of Fuh (2004) states that there exists a $C_{1}, \alpha_{1}>0$, and $\theta_{1}^{*}>0$, such that

$$
\left|\eta_{\theta}(y ; x, b)\right| \leq C_{1} \mathrm{e}^{-\alpha_{1} b}
$$

for all $x, y \in \mathcal{X}, b$ and all $\theta \in\left[0, \theta_{1}^{*}\right]$. Also, by making use of the requirement that $\Theta$ contains an open interval around 0, Lemma 14 of Fuh (2004) (which states that there exists an $\alpha^{*}>0$ such that, for any $\alpha \in\left[0, \alpha^{*}\right], \mathrm{E}_{v}^{\theta} \exp \left\{\alpha \xi_{1}\right\}<\infty$ implies that $\left.\mathrm{E}_{\pi_{+}}^{\theta} \exp \left\{\alpha S_{\tau_{+}}\right\}<\infty\right)$, and Wald's likelihood ratio identity for Markov random walks (see Fuh (2004)), we can show that there exists a $C_{2}, \alpha_{2}>0$, and $\theta_{2}^{*}>0$ such that $\mathrm{E}_{\pi_{+}}^{\theta} \exp \left\{\alpha_{2} S_{\tau_{+}} \mathbf{1}_{\left\{X_{\tau_{+}} \in A\right\}}\right\} \leq C_{2}$ for all $\theta \in\left[0, \theta_{2}^{*}\right]$, so that, for any $y \in \mathcal{X}$ and $A \in \mathcal{A}$,

$$
\mathrm{P}_{y}^{\theta}\left\{X_{\tau_{+}} \in A, S_{\tau_{+}}>s\right\} \leq C_{2} \exp \left\{-\alpha_{2} s\right\} \quad \text { for all } \theta \in\left[0, \theta_{2}^{*}\right] \text { and } s \geq 0 .
$$

Therefore, by (3.4) and (3.5), letting $\alpha_{3}=\min \left\{\alpha_{1}, \alpha_{2}\right\}$ and $\theta^{*}=\min \left\{\theta_{1}^{*}, \theta_{2}^{*}\right\}$, there exists a $C_{3}$ such that, for any $x \in \mathcal{X}$ and $A \in \mathcal{A}$,

$$
\begin{aligned}
& \mid \int_{X}\left(\eta_{\theta}(y ; x, b) \mathrm{P}_{y}^{\theta}\left\{X_{\tau_{+}} \in A, S_{\tau_{+}}>s\right\}\right. \\
& \left.\quad+\frac{\pi_{+}(y) \mathrm{E}_{\pi_{+}}^{\theta} S_{\tau_{+}}^{2}}{2\left(\mathrm{E}_{\pi_{+}}^{\theta} S_{\tau_{+}}\right)^{2}} \mathrm{P}_{y}^{\theta}\left\{X_{\tau_{+}} \in A, S_{\tau_{+}}>b+s\right\}\right) \mathrm{d} M(y) \mid \leq C_{3} \exp \left\{-\alpha_{3}(b+s)\right\}
\end{aligned}
$$

for all $s, b \geq 0$ and $\theta \in\left[0, \theta^{*}\right]$. As, by Theorem 1 of Fuh (2004), the last term on the right-hand side of (3.3) can be rewritten as

$$
\begin{aligned}
& \left|\int_{X} \int_{[0, b / 2)} \mathrm{P}_{y}^{\theta}\left\{X_{\tau_{+}} \in A, S_{\tau_{+}} \in b+s-\mathrm{d} t\right\} \eta_{\theta}(y ; x, t) \mathrm{d} M(y)\right| \\
& \quad \leq C_{1} \mathrm{P}_{\pi_{+}}^{\theta}\left\{X_{\tau_{+}} \in A, S_{\tau_{+}} \geq s+\frac{b}{2}\right\} \\
& \quad \leq C_{1} C_{2} \exp \left\{-\alpha_{2}\left(s+\frac{b}{2}\right)\right\}
\end{aligned}
$$

and

$$
\begin{aligned}
& \left|\int_{X} \int_{[b / 2, b)} \mathrm{P}_{y}^{\theta}\left\{X_{\tau_{+}} \in A, S_{\tau_{+}} \in+s-\mathrm{d} t\right\} \eta_{\theta}(y ; x, t) \mathrm{d} M(y)\right| \\
& \quad \leq C_{1} \exp \left\{\frac{-\alpha_{1} b}{2}\right\} \mathrm{P}_{\pi_{+}}^{\theta}\left\{X_{\tau_{+}} \in A, S_{\tau_{+}} \geq s\right\} \\
& \quad \leq C_{1} C_{2} \exp \left\{\frac{-\alpha_{1} b}{2}+\alpha_{2} s\right\}
\end{aligned}
$$


then there exists an $\alpha_{4}>0$ and a $C_{4}$ such that the integral over $[0, b]$ is bounded by $C_{4} \exp \left\{-\alpha_{4}(b+s)\right\}$ for all $x \in \mathcal{X}, b, s \geq 0$, and $\theta \in\left[0, \theta^{*}\right]$. Thus, by (3.3) there exists an $\alpha_{5}>0$ and a $C_{5}$ such that, for any $x \in \mathcal{X}$ and $A \in \mathcal{A}$,

$$
\left|J_{1}\right| \leq C_{5} \exp \left\{-\alpha_{5}(b+s)\right\}
$$

for all $b, s \geq 0$ and $\theta \in\left[0, \theta^{*}\right]$.

For $J_{2}$, since $\mathrm{E}_{\pi_{+}}^{\theta} S_{\tau_{+}}$is bounded below for $\theta \geq 0$, we have, by Theorem 1 of Fuh (2004),

$$
\left|J_{2}\right| \leq \int_{\chi} \int_{-\infty}^{0} C_{2} \exp \left\{-\alpha_{2}(b+s-t)\right\} \frac{\mathrm{d} t}{C_{6}} \pi_{+}(y) \mathrm{d} M(y)=C_{7} \exp \left\{-\alpha_{2}(b+s)\right\} .
$$

Combining (3.2), (3.6), and (3.7), we complete the proof.

Recall the definitions of $\theta_{1}$ and $\theta_{0}$ given in the paragraph before Theorem 2.1, and let $\Delta=\theta_{1}-\theta_{0}$. By making use of the fact that $\mathrm{E}_{x}^{\theta} R_{b}^{a}=a \int_{0}^{\infty} s^{a-1} \mathrm{P}_{x}^{\theta}\left\{X_{\tau_{b}} \in \mathcal{X}, R_{b}>s\right\} \mathrm{d} s$ and Theorem 3.1, we obtain the following result.

Corollary 3.1. Assume that the conditions of Theorem 3.1 hold. Then, for any $a>0$, there exists an $r>0, \theta^{*}>0$, and $C$ such that, for any $x \in \mathcal{X}$,

$$
\left|\mathrm{E}_{x}^{\theta} R_{b}^{a}-\mathrm{E}_{\pi_{+}}^{\theta} R_{\infty}^{a}\right| \leq C \mathrm{e}^{-r b}
$$

for all $b \geq 0$ and $\theta \in\left[0, \theta^{*}\right]$. Also, there exists an $r>0, \theta^{*}>0$, and $C$ such that, for any $x \in \mathcal{X}$,

$$
\left|\mathrm{E}_{x}^{\theta_{1}}\left(\mathrm{e}^{-\Delta R_{b}}\right)-\mathrm{E}_{\pi_{+}}^{\theta_{1}}\left(\mathrm{e}^{-\Delta R_{\infty}}\right)\right| \leq C \Delta \mathrm{e}^{-r b}
$$

for all $b \geq 0$ and $\theta_{1} \in\left[0, \theta^{*}\right]$.

\section{The covariance between the first passage time and the overshoot}

For given $\theta \in\left(0, \theta^{*}\right]$ and for any $u>0, x \in \mathcal{X}$, and $A \in \mathcal{A}$, let

$$
\begin{gathered}
Q_{x}^{\theta}(A, u ; b)=\sum_{n=0}^{\infty} \mathrm{P}_{x}^{\theta}\left\{\tau_{b}>n, X_{n} \in A, S_{n}>b-u\right\}, \\
Q_{\pi_{+}}^{\theta}(A, u ; \infty)=\left(\mathrm{E}_{\pi_{+}}^{\theta} S_{\tau_{+}}\right)^{-1} \int_{-u}^{0} \int_{X}\left\{\int_{A} \widehat{U}_{-}^{\theta}(z, y ;(s, 0)) \mathrm{d} M(y)\right\} \mathrm{d} \pi_{+}(z) \mathrm{d} s,
\end{gathered}
$$

where $\widehat{U}_{-}^{\theta}(z, y ;(s, 0))$ is defined as $\widehat{U}_{-}(z, y ;(s, 0))$ in Section 3 for the Markov random walk $\left\{\left(X_{n}^{\theta}, S_{n}^{\theta}\right), n \geq 0\right\}$. In order to study the rate of convergence for the covariance between the first passage time and the overshoot, we first give the following two lemmas. Lemma 4.1 generalizes Lemma 3.1 of Fuh and Lai (2001) in order to obtain the rate of convergence.

Lemma 4.1. Let $\left\{\left(X_{n}, S_{n}\right), n \geq 0\right\}$ be a Markov random walk satisfying Assumptions 2.1-2.6 and denote by $\left\{\left(X_{n}^{\theta}, S_{n}^{\theta}\right), n \geq 0\right\}$ the Markov random walk induced by (2.13) with $\mathrm{E}_{\pi}^{\theta} S_{1}>0$. Then there exists $a C, r_{1}>0$, and $\theta^{*}>0$ such that, for any $x \in \mathcal{X}$ and $A \in \mathcal{A}$,

$$
\left|Q_{x}^{\theta}(A, u ; b)-Q_{\pi_{+}}^{\theta}(A, u ; \infty)\right| \leq C \mathrm{e}^{-r_{1}(b-u)}
$$

for all $b>0,0 \leq u \leq b$, and $\theta \in\left(0, \theta^{*}\right]$. 
Proof. First note that, for any $x, z \in \mathcal{X}$,

$$
\begin{aligned}
\mathrm{P}_{x}^{\theta}\left\{\tau_{b}>n, X_{n} \in A,\right. & \left.S_{n}>b-u\right\} \\
= & \sum_{k=0}^{n} \int_{(b-u, b)} \mathrm{P}_{x}^{\theta}\left\{S_{i}<S_{k} \text { for all } i<k, S_{k} \in \mathrm{d} t, S_{j} \leq S_{k} \text { for all } k<j \leq n,\right. \\
& \left.S_{n}-S_{k}>b-u-t, X_{n} \in A\right\} \\
= & \sum_{k=0}^{n} \int_{(0, u)} \int_{X} \mathrm{P}_{x}^{\theta}\left\{S_{i}<S_{k} \text { for all } i<k, S_{k} \in b-\mathrm{d} s, X_{k} \in \mathrm{d} z\right\} \\
& \times \mathrm{P}_{z}^{\theta}\left\{\tau_{+}>n-k, S_{n-k}>s-u, X_{n-k} \in A\right\} .
\end{aligned}
$$

Recall that $\tau_{+}^{m}$ is the $m$ th ascending ladder epoch. Then

$\sum_{k=0}^{\infty} \mathrm{P}_{x}^{\theta}\left\{S_{i}<S_{k}\right.$ for all $\left.i<k, S_{k} \in b-\mathrm{d} s, X_{k} \in \mathrm{d} z\right\}=\sum_{m=0}^{\infty} \mathrm{P}_{x}^{\theta}\left\{X_{\tau_{+}^{m}} \in \mathrm{d} z, S_{\tau_{+}^{m}} \in b-\mathrm{d} s\right\}$.

For given $A \in \mathcal{X}$ and $B \in \mathscr{B}((-\infty, 0))$, as shown by Fuh and Lai (1998, p. 576), $\sum_{n=0}^{\infty} \mathrm{P}_{z}^{\theta}\left\{\tau_{+}>n, X_{n} \in A, S_{n} \in B\right\}=\int_{A} \widehat{U}_{-}^{\theta}(z, y ; B) \mathrm{d} M(y)$. Then

$$
\begin{aligned}
Q_{x}^{\theta}(A, u ; b) & =\sum_{n=0}^{\infty} \mathrm{P}_{z}^{\theta}\left\{\tau_{b}>n, X_{n} \in A, S_{n}>b-u\right\} \\
& =\sum_{j=0}^{\infty} \int_{(0, u)} \int_{X} \sum_{m=0}^{\infty} \mathrm{P}_{x}^{\theta}\left\{S_{\tau_{+}^{m}} \in b-\mathrm{d} s, X_{\tau_{+}^{m}} \in \mathrm{d} z\right\} \\
& \times \mathrm{P}_{z}^{\theta}\left\{\tau_{+}>j, S_{j}>s-u, X_{j} \in A\right\} \\
& =\int_{(0, u)} \int_{x}\left\{\int_{A} \widehat{U}_{-}^{\theta}(z, y ;(s-u, 0)) \mathrm{d} M(y)\right\} U_{x,+}^{\theta}(z, b-\mathrm{d} s) \mathrm{d} \pi_{+}(z),
\end{aligned}
$$

where $U_{x,+}^{\theta}(z, u):=\sum_{m=0}^{\infty} \mathrm{P}_{x}^{\theta}\left\{X_{\tau_{+}^{m}} \in \mathrm{d} z, S_{\tau_{+}^{m}} \leq u\right\}$. Therefore,

$$
\begin{aligned}
& Q_{x}^{\theta}(A, u ; b)-Q_{\pi_{+}}^{\theta}(A, u ; \infty) \\
& =\int_{X} \int_{0}^{u}\left\{\int_{A} \widehat{U}_{-}^{\theta}(z, y ;(-t, 0)) \mathrm{d} M(y)\right\} \\
& \quad \times\left(U_{x,+}^{\theta}(z, b-u+d t)-U_{x,+}^{\theta}(z, b-u)-\frac{\mathrm{d} t}{\mathrm{E}_{\pi_{+}}^{\theta} S_{\tau_{+}}}\right) \mathrm{d} \pi_{+}(z) \\
& =\int_{X}\left\{\int_{A} \widehat{U}_{-}^{\theta}(z, y ;(-u, 0)) \mathrm{d} M(y)\right\} \\
& \quad \times\left(U_{x,+}^{\theta}(z, b)-U_{x,+}^{\theta}(z, b-u)-\frac{u}{\mathrm{E}_{\pi_{+}}^{\theta} S_{\tau_{+}}}\right) \mathrm{d} \pi_{+}(z) \\
& \quad \int_{x} \int_{0}^{u}\left(U_{x,+}^{\theta}(z, b-u+t)-U_{x,+}^{\theta}(z, b-u)-\frac{t}{\mathrm{E}_{\pi_{+}}^{\theta} S_{\tau_{+}}}\right) \\
& \quad \times \mathrm{d}\left\{\int_{A} \widehat{U}_{-}^{\theta}(z, y ;(-t, 0)) \mathrm{d} M(y)\right\} \mathrm{d} \pi_{+}(z) .
\end{aligned}
$$


Combining these representations with the rate of convergence for the Markov renewal theorem (see (2.7) in Theorem 1 of Fuh (2004)), we have $\left|U_{x,+}^{\theta}(z, b)-U_{x,+}^{\theta}(z, b-u)-u / \mathrm{E}_{\pi_{+}}^{\theta} S_{\tau_{+}}\right|=$ $o\left(\mathrm{e}^{-r_{1}(b-u)}\right)$ and $\left|U_{x,+}^{\theta}(z, b-u+t)-U_{x,+}^{\theta}(z, b-u)-t / \mathrm{E}_{\pi_{+}}^{\theta} S_{\tau_{+}}\right|=o\left(\mathrm{e}^{-r_{1}(b-u)}\right)$. This yields (4.2).

Lemma 4.2. Let $\left\{\left(X_{n}, S_{n}\right), n \geq 0\right\}$ be a Markov random walk satisfying Assumptions 2.1-2.6 and denote by $\left\{\left(X_{n}^{\theta}, S_{n}^{\theta}\right), n \geq 0\right\}$ the Markov random walk induced by (2.13) with $\mathrm{E}_{\pi}^{\theta} S_{1}>0$. Then there exists $a \theta^{*}>0$ such that, for all $a>0$ and $\theta \in\left(0, \theta^{*}\right)$,

$$
\sup _{b \geq 0} \mathrm{E}_{\pi}^{\theta} R_{b}^{a} \leq \frac{a+2}{a+1} \frac{\mathrm{E}_{\pi}^{\theta}\left(\xi_{1}^{+}\right)^{a+1}}{\mathrm{E}_{\pi}^{\theta} \xi_{1}} .
$$

When the initial distribution of $X_{0}$ is $v$, (4.3) implies that there exists a constant $K>0$ such that $\sup _{b \geq 0} \mathrm{E}_{v}^{\theta} R_{b}^{a} \leq(a+2) \mathrm{E}_{\pi}^{\theta}\left(\xi_{1}^{+}\right)^{a+1} /\left((a+1) \mathrm{E}_{\pi}^{\theta} \xi_{1}\right)+K$.

In the case of simple random walks, the upper bound (4.3) was given in Theorem 3 of Lorden (1970) by pathwise integration. In the case of Markov random walks, the upper bound (4.3) was given by Fuh (2004) when $a=1$. Here we generalize it for all $a>0$ in Lemma 4.2, which will be used in the proof of Theorem 4.1. Since the proof of Lemma 4.2 is a simple consequence of Lemma 2 of Fuh (2004) using Theorem 3 of Lorden (1970), we omit it here.

Theorem 4.1. Let $\left\{\left(X_{n}, S_{n}\right), n \geq 0\right\}$ be a Markov random walk satisfying Assumptions 2.1-2.6 and denote by $\left\{\left(X_{n}^{\theta}, S_{n}^{\theta}\right), n \geq 0\right\}$ the Markov random walk induced by (2.13) with $\mathrm{E}_{\pi}^{\theta} S_{1}>0$. Let $\psi^{a}(\theta, v)=\mathrm{E}_{\pi_{+}}^{\theta} R_{v}^{a}-\mathrm{E}_{\pi_{+}}^{\theta} R_{\infty}^{a}$ and define

$$
C^{a}(\theta)=\frac{1}{\mathrm{E}_{\pi_{+}}^{\theta} S_{\tau_{+}}} \int_{0}^{\infty} \psi^{a}(\theta, v) \int_{X} \int_{X} \widehat{U}_{-}^{\theta}(z, y ;(-v, 0)) \mathrm{d} M(y) \mathrm{d} \pi_{+}(z) \mathrm{d} v .
$$

Then there exists a $C, r>0$, and $\theta^{*}>0$ such that, for any $x \in \mathcal{X}$,

$$
\left|\operatorname{cov}_{x}^{\theta}\left(\tau_{b}, R_{b}^{a}\right)-C^{a}(\theta)\right| \leq\left(\mathrm{E}_{\pi_{+}}^{\theta} S_{\tau_{+}}\right)^{-1} C \mathrm{e}^{-r b}
$$

for all $b>0$ and $\theta \in\left(0, \theta^{*}\right]$, where $\operatorname{cov}_{x}^{\theta}(\cdot, \cdot)$ denotes the covariance of two random variables.

Remark. The convergence of $\operatorname{cov}_{x}^{\theta}\left(\tau_{b}, R_{b}^{a}\right)$ to $C^{a}(\theta)$ can be found in (3.18) of Fuh and Lai (2001). Theorem 4.1 establishes exponential rate of convergence uniformly in $x \in \mathcal{X}$. The constant term, $\left(\mathrm{E}_{\pi_{+}}^{\theta} S_{\tau_{+}}\right)^{-1}$, on the right-hand side of (4.4) comes from Wald's equation for Markov random walks.

Proof of Theorem 4.1. First, we note that it is sufficient to show that there exists a $C_{1}, C_{2}$, $r>0$, and $\theta^{*}>0$ such that

$$
\left|\operatorname{cov}_{x}^{\theta}\left(\tau_{b}, R_{b}^{a}\right)-C^{a}(\theta)\right| \leq\left(\mathrm{E}_{\pi_{+}}^{\theta} S_{\tau_{+}}\right)^{-1}\left(A_{1} b+A_{2}\right) \mathrm{e}^{-r b}
$$

for all $b>0$ and $\theta \in\left(0, \theta^{*}\right]$.

To prove (4.5), we assume that $\theta>0$ is fixed, then

$$
\operatorname{cov}_{x}^{\theta}\left(\tau_{b}, R_{b}^{a}\right)=\int_{z \in \mathcal{X}} \int_{0}^{\infty}\left\{\mathrm{E}_{z}^{\theta} R_{v}^{a}-\mathrm{E}_{z}^{\theta} R_{b}^{a}\right\} Q_{x}^{\theta}(\mathrm{d} z, \mathrm{~d} v ; b) .
$$


Next, (3.18) of Fuh and Lai (2001) shows (for the case in which $a=1$, but its generalization to $a>0$ is straightforward) that, as $b \rightarrow \infty$,

$$
\operatorname{cov}_{x}^{\theta}\left(\tau_{b}, R_{b}^{a}\right) \rightarrow C^{a}(\theta):=\int_{0}^{\infty} \psi^{a}(\theta, v) Q_{\pi_{+}}^{\theta}(\mathrm{d} v ; \infty),
$$

where $Q_{\pi_{+}}^{\theta}(\mathrm{d} v ; \infty)=\int_{z \in \mathcal{X}} Q_{\pi_{+}}^{\theta}(\mathrm{d} z, \mathrm{~d} v ; \infty)$.

Let $Q_{x}^{\theta}(\mathrm{d} v ; b)=\int_{X} Q_{x}^{\theta}(\mathrm{d} z, \mathrm{~d} v ; b)$. From (4.6) and (4.7), and noting that

$$
\int_{[0, \infty)} Q_{x}^{\theta}(\mathrm{d} v ; b)=\mathrm{E}_{x}^{\theta} \tau_{b}
$$

we have

$$
\begin{aligned}
\operatorname{cov}_{x}^{\theta}\left(\tau_{b}, R_{b}^{a}\right)-C^{a}(\theta) & \\
= & \int_{0}^{\infty}\left(\mathrm{E}_{\pi_{+}}^{\theta} R_{v}^{a}-\mathrm{E}_{\pi_{+}}^{\theta} R_{\infty}^{a}\right)\left[Q_{x}^{\theta}(\mathrm{d} v ; b)-Q_{\pi_{+}}^{\theta}(\mathrm{d} v ; \infty)\right]+\left(\mathrm{E}_{\pi_{+}}^{\theta} R_{\infty}^{a}-\mathrm{E}_{\pi_{+}}^{\theta} R_{b}^{a}\right) \mathrm{E}_{x}^{\theta} \tau_{b} \\
& +\int_{z \in \mathcal{X}} \int_{0}^{\infty}\left[\left(\mathrm{E}_{z}^{\theta} R_{v}^{a}-\mathrm{E}_{\pi_{+}}^{\theta} R_{v}^{a}\right)-\left(\mathrm{E}_{z}^{\theta} R_{b}^{a}-\mathrm{E}_{\pi_{+}}^{\theta} R_{\infty}^{a}\right)\right] Q_{x}^{\theta}(\mathrm{d} z, \mathrm{~d} v ; b)
\end{aligned}
$$

We show that the second term on the right-hand side of (4.8) satisfies (4.5) as follows. First, by the assumption of the continuity of $\mathrm{E}_{\pi_{+}}^{\theta} S_{\tau_{+}}$in $\theta$, we can choose $C>0$ and $\theta^{*}>0$ such that, for all $\theta \in\left[0, \theta^{*}\right], \mathrm{E}_{\pi_{+}}^{\theta} S_{\tau_{+}} \geq C^{-1}$. Next, by Theorem 1 of Fuh (2004), there exists a $u>0$ such that

$$
\left|U_{v,+}^{\theta}(s)-\frac{s+\mathrm{E}_{\pi_{+}}^{\theta} R_{\infty}}{\mathrm{E}_{\pi_{+}}^{\theta} S_{\tau_{+}}}\right| \leq C \mathrm{e}^{-u s},
$$

where $U_{v,+}^{\theta}(s)=\sum_{n=0}^{\infty} \mathrm{P}_{v}^{\theta}\left\{X_{\tau_{+}^{n}} \in \mathcal{X}, S_{\tau_{+}^{n}} \in[-\infty, s]\right\}$. By making use of Wald's equation for Markov random walks, Lemma 4.2, and Corollary 3.1, we obtain, for any $x \in \mathcal{X}$,

$$
\left|\left(\mathrm{E}_{\pi_{+}}^{\theta} R_{b}^{a}-\mathrm{E}_{\pi_{+}}^{\theta} R_{\infty}^{a}\right) \mathrm{E}_{x}^{\theta} \tau_{b}\right| \leq C \mathrm{e}^{-u b} \frac{b+C}{\mathrm{E}_{\pi_{+}}^{\theta} S_{\tau_{+}}}
$$

for all $b>0$ and $\theta \in\left(0, \theta^{*}\right]$.

Next we show that the first term on the right-hand side of (4.8) satisfies (4.5). For this purpose, we split the interval of the first integral in (4.8) into two subintervals, i.e. [0,b/2) and $[b / 2, \infty)$. For the integral over $[0, b / 2]$, we write

$$
\begin{aligned}
\int_{[0, b / 2)}\left\{\mathrm{E}_{\pi_{+}}^{\theta} R_{v}^{a}-\mathrm{E}_{\pi_{+}}^{\theta} R_{\infty}^{a}\right\}\left[Q_{x}^{\theta}(\mathrm{d} v ; b)-Q_{\pi_{+}}^{\theta}(\mathrm{d} v ; \infty)\right] \\
=\left\{\mathrm{E}_{\pi_{+}}^{\theta} R_{v}^{a}-\mathrm{E}_{\pi_{+}}^{\theta} R_{\infty}^{a}\right\}\left[Q_{x}^{\theta}\left(\frac{b}{2} ; b\right)-Q_{\pi_{+}}^{\theta}\left(\frac{b}{2} ; \infty\right)\right] \\
-\int_{[0, b / 2)}\left[Q_{x}^{\theta}\left(\frac{b}{2} ; b\right)-Q_{\pi_{+}}^{\theta}\left(\frac{b}{2} ; \infty\right)\right] \mathrm{d}\left(\mathrm{E}_{\pi_{+}}^{\theta} R_{v}^{a}\right),
\end{aligned}
$$

where we have used $Q_{x}^{\theta}\left(0^{-} ; b\right)=0=Q_{\pi_{+}}^{\theta}\left(0^{-} ; \infty\right)$. However, by Corollary 3.1 and Lemma 4.1, we have

$$
\left|\left\{\mathrm{E}_{\pi_{+}}^{\theta} R_{v}^{a}-\mathrm{E}_{\pi_{+}}^{\theta} R_{\infty}^{a}\right\}\left[Q_{x}^{\theta}\left(\frac{b}{2} ; b\right)-Q_{\pi_{+}}^{\theta}\left(\frac{b}{2} ; \infty\right)\right]\right| \leq 4 C^{2}\left(\mathrm{E}_{\pi_{+}}^{\theta} S_{\tau_{+}}\right)^{-1} \mathrm{e}^{-s b} .
$$


Note that $\int_{0}^{v} a \mathrm{E}_{\pi_{+}}^{\theta} R_{t}^{a-1} \mathrm{~d} t=\left(\mathrm{E}_{\pi_{+}}^{\theta} S_{\tau_{+}}^{a}\right) U_{\pi_{+},+}^{\theta}(v)-\mathrm{E}_{\pi_{+}}^{\theta} R_{v}^{a}$, and this implies that, for any $x \in \mathcal{X}$,

$$
\begin{aligned}
& \left|\int_{[0, b / 2)}\left[Q_{x}^{\theta}(\mathrm{d} v ; b)-Q_{\pi_{+}}^{\theta}(\mathrm{d} v ; \infty)\right] \mathrm{d}\left(\mathrm{E}_{\pi_{+}}^{\theta} R_{v}^{a}\right)\right| \\
& \quad \leq\left(\sup _{0 \leq v<b / 2}\left[Q_{x}^{\theta}(v ; b)-Q_{\pi_{+}}^{\theta}(v ; \infty)\right]\right)\left(\left(\mathrm{E}_{\pi_{+}}^{\theta} S_{\tau_{+}}^{a}\right) U_{\pi_{+},+}^{\theta}\left(\frac{b}{2}\right)+\int_{0}^{b / 2} a \mathrm{E}_{\pi_{+}}^{\theta} R_{v}^{a-1} \mathrm{~d} v\right) \\
& \quad \leq\left(4 C^{2}\left(\mathrm{E}_{\pi_{+}}^{\theta} S_{\tau_{+}}\right)^{-1} \mathrm{e}^{-s b}\right)\left[2\left(\mathrm{E}_{\pi_{+}}^{\theta} S_{\tau_{+}}^{a}\right) U_{\pi_{+},+}^{\theta}\left(\frac{b}{2}\right)\right] \\
& \quad \leq 8 C^{3}\left(\mathrm{E}_{\pi_{+}}^{\theta} S_{\tau_{+}}\right)^{-1}\left[\frac{b}{2}+C\right] \mathrm{e}^{-s b / 2} .
\end{aligned}
$$

Combining (4.11) and (4.12) shows that (4.10) satisfies (4.5).

Finally, for the integral over $[b / 2, \infty)$, we use Corollary 3.1 to write

$$
\begin{aligned}
& \left|\int_{[b / 2, \infty)}\left\{\mathrm{E}_{\pi_{+}}^{\theta} R_{v}^{a}-\mathrm{E}_{\pi_{+}}^{\theta} R_{\infty}^{a}\right\}\left[Q_{x}^{\theta}(\mathrm{d} v ; b)-Q_{\pi_{+}}^{\theta}(\mathrm{d} v ; \infty)\right]\right| \\
& \quad \leq \int_{[b / 2, \infty)} C \mathrm{e}^{-s v} Q_{x}^{\theta}(\mathrm{d} s ; b)+\int_{[b / 2, \infty)} C \mathrm{e}^{-s v} Q_{\pi_{+}}^{\theta}(\mathrm{d} v ; \infty) .
\end{aligned}
$$

It is easy to see that the last two integrals satisfy (4.5). Using

$$
\int_{[0, \infty)} Q_{x}^{\theta}(\mathrm{d} v ; b)=\mathrm{E}_{\pi_{+}}^{\theta} \tau_{b} \leq\left(\mathrm{E}_{\pi_{+}}^{\theta} S_{\tau_{+}}\right)^{-1}(b+C)
$$

and Lemma 3.1 of Fuh and Lai (2001), we can prove that the first term of (4.8) satisfies (4.5).

It remains to show that the third term on the right-hand side of (4.8) satisfies (4.5). By Corollary 3.1, we find that, for any $a>0$, there exists a $C, r>0$, and $\theta^{*}>0$ such that

$$
\left|\mathrm{E}_{z}^{\theta} R_{v}^{a}-\mathrm{E}_{\pi_{+}}^{\theta} R_{v}^{a}\right| \leq\left|\mathrm{E}_{z}^{\theta} R_{v}^{a}-\mathrm{E}_{\pi_{+}}^{\theta} R_{\infty}^{a}\right|+\left|\mathrm{E}_{\pi_{+}}^{\theta} R_{\infty}^{a}-\mathrm{E}_{\pi_{+}}^{\theta} R_{v}^{a}\right| \leq 2 C \mathrm{e}^{-r v}
$$

for all $v \geq 0, \theta \in\left[0, \theta^{*}\right]$, and uniformly for $z \in \mathcal{X}$. Using the same argument as used for (4.13) and (4.14), we have, for any $x \in \mathcal{X}$,

$$
\left|\int_{z \in \mathcal{X}} \int_{0}^{\infty}\left[\left(\mathrm{E}_{z}^{\theta} R_{v}^{a}-\mathrm{E}_{\pi_{+}}^{\theta} R_{v}^{a}\right)-\left(\mathrm{E}_{z}^{\theta} R_{b}^{a}-\mathrm{E}_{\pi_{+}}^{\theta} R_{\infty}^{a}\right)\right] Q_{x}^{\theta}(\mathrm{d} z, \mathrm{~d} v ; b)\right| \leq 3 C \mathrm{e}^{-r b} .
$$

This completes the proof.

Let

$$
\tilde{C}\left(\theta_{1}\right)=\int_{0}^{\infty}\left[\mathrm{E}_{\pi_{+}}^{\theta_{1}}\left(\mathrm{e}^{-\Delta R_{v}}\right)-\mathrm{E}_{\pi_{+}}^{\theta_{1}}\left(\mathrm{e}^{-\Delta R_{\infty}}\right)\right] Q_{\pi_{+}}^{\theta_{1}}(\mathrm{~d} v ; \infty) .
$$

Theorem 4.2. Let $\left\{\left(X_{n}, S_{n}\right), n \geq 0\right\}$ be a Markov random walk satisfying Assumptions 2.1-2.6 and denote by $\left\{\left(X_{n}^{\theta_{1}}, S_{n}^{\theta_{1}}\right), n \geq 0\right\}$ the Markov random walk induced by (2.13) with $\mathrm{E}_{\pi}^{\theta_{1}} S_{1}>0$. Then there exists an $A, r>0$, and $\theta^{*}>0$ such that, for any $x \in \mathcal{X}$,

$$
\left|\operatorname{cov}_{x}^{\theta_{1}}\left(\tau_{b}, \mathrm{e}^{-\Delta R_{b}}\right)-\tilde{C}\left(\theta_{1}\right)\right| \leq A \mathrm{e}^{-r b}
$$

for all $b>0$ and $\theta_{1} \in\left(0, \theta^{*}\right]$. 
Proof. Using the same method as above to derive (4.8), we have, for any $x \in \mathcal{X}$,

$$
\begin{aligned}
& \operatorname{cov}_{x}^{\theta_{1}}\left(\tau_{b}, \mathrm{e}^{-\Delta R_{b}}\right)-\tilde{C}\left(\theta_{1}\right) \\
& =\int_{0}^{\infty}\left[\mathrm{E}_{\pi_{+}}^{\theta_{1}}\left(\mathrm{e}^{-\Delta R_{v}}\right)-\mathrm{E}_{\pi_{+}}^{\theta_{1}}\left(\mathrm{e}^{-\Delta R_{\infty}}\right)\right]\left[Q_{x}^{\theta_{1}}(\mathrm{~d} v ; b)-Q_{\pi_{+}}^{\theta_{1}}(\mathrm{~d} v ; \infty)\right] \\
& \quad+\left[\mathrm{E}_{\pi_{+}}^{\theta_{1}}\left(\mathrm{e}^{-\Delta R_{\infty}}\right)-\mathrm{E}_{\pi_{+}}^{\theta_{1}}\left(\mathrm{e}^{-\Delta R_{b}}\right)\right] \mathrm{E}_{x}^{\theta_{1}} \tau_{b} \\
& \quad+\int_{z \in \mathcal{X}} \int_{0}^{\infty}\left[\left(\mathrm{E}_{z}^{\theta_{1}}\left(\mathrm{e}^{-\Delta R_{v}}\right)-\mathrm{E}_{\pi_{+}}^{\theta_{1}}\left(\mathrm{e}^{-\Delta R_{v}}\right)\right)-\left(\mathrm{E}_{z}^{\theta_{1}}\left(\mathrm{e}^{-\Delta R_{b}}\right)-\mathrm{E}_{\pi_{+}}^{\theta_{1}}\left(\mathrm{e}^{-\Delta R_{\infty}}\right)\right)\right] \\
& \quad \times Q_{x}^{\theta_{1}}(\mathrm{~d} z, \mathrm{~d} v ; b) .
\end{aligned}
$$

The rest of the proof is the same as that of Theorem 4.1.

Theorem 4.3. Let $\left\{\left(X_{n}, S_{n}\right), n \geq 0\right\}$ be a Markov random walk satisfying Assumptions 2.1-2.6 and denote by $\left\{\left(X_{n}^{\theta}, S_{n}^{\theta}\right), n \geq 0\right\}$ the Markov random walk induced by (2.13) with $\mathrm{E}_{\pi}^{\theta} S_{1}>0$. Then there exists a $\theta^{*}>0$ such that, for any $x \in \mathcal{X}$,

$$
\operatorname{cov}_{x}^{\theta}\left(\tau_{b}, R_{b}^{a}\right)=\frac{1}{\mathrm{E}_{\pi}^{\theta} S_{1}}\left[\mathrm{E}_{x}^{\theta} R_{b}^{a+1}-\left(\mathrm{E}_{x}^{\theta} R_{b}\right)\left(\mathrm{E}_{x}^{\theta} R_{b}^{a}\right)-\dot{\mathrm{E}}_{x}^{\theta} R_{b}+K_{\theta}\right]
$$

for all $\theta \in\left(0, \theta^{*}\right]$, where

$$
K_{\theta}=\mathrm{E}_{x}^{\theta}\left(\frac{\dot{r}\left(X_{\tau_{b}} ; \theta\right)}{r\left(X_{\tau_{b}} ; \theta\right)}-\frac{\dot{r}(x ; \theta)}{r(x ; \theta)}\right)-\left(\mathrm{E}_{x}^{\theta} \dot{r}\left(X_{\tau_{b}} ; \theta\right)-\dot{r}(x ; \theta)\right)\left(\mathrm{E}_{x}^{\theta} R_{b}^{a}\right) .
$$

Proof. For given $\theta>0$, by Wald's likelihood ratio identity for Markov random walks, we have

$$
\mathrm{E}_{x}^{\theta} R_{b}^{a}=\mathrm{E}_{x}\left\{R_{b}^{a} \exp \left[\theta S_{\tau_{b}}-\tau_{b} \Lambda(\theta)\right] \frac{r\left(X_{\tau_{b}} ; \theta\right)}{r(x ; \theta)}\right\} .
$$

Under Assumptions 2.1-2.6, (5.31) in Lemma 5.4 implies that $|\dot{r}(x ; 0)| \leq\left|\mathrm{E}_{x} \xi_{1}-\mu\right|$. By making use of the fact that $\mathrm{E}_{\pi}\left|\mathrm{E}_{x} \xi_{1}-\mu\right|<\infty$ and the continuous differentiability property of $r(x ; \theta)$ (see Fuh (2004, Proposition 1)), we show that there exists a $\delta>0$ such that, for $|\theta| \leq \delta, \mathrm{E}_{x} \dot{r}\left(X_{1} ; \theta\right)<\infty$ uniformly for all $x \in \mathcal{X}$. Now using the dominated convergence theorem to interchange differentiation with expectation, we obtain

$$
\begin{aligned}
\dot{\mathrm{E}}_{x}^{\theta} R_{b}^{a}= & \mathrm{E}_{x}\left\{R_{b}^{a} \exp \left[\theta S_{\tau_{b}}-\tau_{b} \Lambda(\theta)\right] \frac{r\left(X_{\tau_{b}} ; \theta\right)}{r(x ; \theta)}\left(S_{\tau_{b}}-\tau_{b} \dot{\Lambda}(\theta)\right)\right. \\
& \left.+R_{b}^{a} \exp \left[\theta S_{\tau_{b}}-\tau_{b} \Lambda(\theta)\right] \frac{\dot{r}\left(X_{\tau_{b}} ; \theta\right) r(x ; \theta)-r\left(X_{\tau_{b}} ; \theta\right) \dot{r}(x ; \theta)}{r^{2}(x ; \theta)}\right\} \\
= & \mathrm{E}_{x}\left\{R_{b}^{a} \exp \left[\theta S_{\tau_{b}}-\tau_{b} \Lambda(\theta)\right] \frac{r\left(X_{\tau_{b}} ; \theta\right)}{r(x ; \theta)}\right. \\
& \left.\times\left(\left(S_{\tau_{b}}-\tau_{b} \dot{\Lambda}(\theta)\right)+\frac{\dot{r}\left(X_{\tau_{b}} ; \theta\right) r(x ; \theta)-r\left(X_{\tau_{b}} ; \theta\right) \dot{r}(x ; \theta)}{r\left(X_{\tau_{b}} ; \theta\right) r(x ; \theta)}\right)\right\} \\
= & \mathrm{E}_{x}^{\theta}\left\{R_{b}^{a}\left(S_{\tau_{b}}-\tau_{b} \mathrm{E}_{\pi}^{\theta} S_{1}+G_{\theta}\right)\right\},
\end{aligned}
$$

where we have set $\mathrm{E}_{\pi}^{\theta} S_{1}=\dot{\Lambda}(\theta)$ and $G_{\theta}=\mathrm{E}_{x}^{\theta}\left(\dot{r}\left(X_{\tau_{b}} ; \theta\right) / r\left(X_{\tau_{b}} ; \theta\right)\right)-\dot{r}(x ; \theta) / r(x ; \theta)$. Making the substitution $S_{\tau_{b}}=b+R_{b}$ in (4.16) and rearranging, we obtain

$$
\mathrm{E}_{x}^{\theta}\left(\tau_{b} R_{b}^{a}\right)=\frac{1}{\mathrm{E}_{\pi}^{\theta} S_{1}}\left[b \mathrm{E}_{x}^{\theta} R_{b}^{a}+\mathrm{E}_{x}^{\theta} R_{b}^{a+1}-\dot{\mathrm{E}}_{x}^{\theta} R_{b}^{a}+G_{\theta}\right] .
$$


By Wald's equation for Markov random walks (see Fuh and Lai (1998, Theorem 1) and Fuh and Zhang (2000)), we have

$$
\left(\mathrm{E}_{x}^{\theta} \tau_{b}\right)\left(\mathrm{E}_{x}^{\theta} R_{b}^{a}\right)=\frac{1}{\mathrm{E}_{\pi}^{\theta} S_{1}}\left(b+\mathrm{E}_{x}^{\theta} R_{b}+G_{\theta}^{\prime}\right)\left(\mathrm{E}_{x}^{\theta} R_{b}^{a}\right),
$$

where $G_{\theta}^{\prime}=\mathrm{E}_{x}^{\theta} \dot{r}\left(X_{\tau_{b}} ; \theta\right)-\dot{r}(x ; \theta)$. Equation (4.15) is obtained by subtracting (4.17) from (4.18). The finiteness of $\dot{r}(x ; \theta)$ for all $x \in \mathcal{X}$ will be given in Lemma 5.4, below.

Corollary 4.1. Assume that the conditions of Theorem 4.3 hold. Then there exists a $C, r>0$, and $\theta^{*}>0$ such that, for any $x \in \mathcal{X}$,

$$
\left|\dot{\mathrm{E}}_{x}^{\theta} R_{b}^{a}-\dot{\mathrm{E}}_{\pi_{+}}^{\theta} R_{\infty}^{a}\right| \leq C \mathrm{e}^{-r b}
$$

for all $b>0$ and $\theta \in\left(0, \theta^{*}\right]$.

Proof. Rearranging (4.15) gives

$$
\dot{\mathrm{E}}_{x}^{\theta} R_{b}=\mathrm{E}_{x}^{\theta} R_{b}^{a+1}-\left(\mathrm{E}_{x}^{\theta} R_{b}\right)\left(\mathrm{E}_{x}^{\theta} R_{b}^{a}\right)+K_{\theta}-\left(\mathrm{E}_{\pi}^{\theta} S_{1}\right) \operatorname{cov}_{x}^{\theta}\left(\tau_{b}, R_{b}^{a}\right) .
$$

Define

$$
g(\theta)=\mathrm{E}_{\pi_{+}}^{\theta} R_{\infty}^{a+1}-\left(\mathrm{E}_{\pi_{+}}^{\theta} R_{\infty}\right)\left(\mathrm{E}_{\pi_{+}}^{\theta} R_{\infty}^{a}\right)+K_{\theta}-\left(\mathrm{E}_{\pi}^{\theta} S_{1}\right) C^{a}(\theta) .
$$

Since $K_{\theta}$ is bounded by Proposition 1 of Fuh (2004), using Corollary 3.1 and Theorem 4.1, it is easy to see that there exists a $C, r>0$, and $\theta^{*}>0$ such that, for any $x \in \mathcal{X}$,

$$
\left|\dot{\mathrm{E}}_{x}^{\theta} R_{b}^{a}-g(\theta)\right| \leq C \mathrm{e}^{-r b}
$$

for all $b>0$ and $\theta \in\left(0, \theta^{*}\right]$.

Now, we have, as $b \rightarrow \infty, \mathrm{E}_{x}^{\theta} R_{b}^{a} \rightarrow \mathrm{E}_{\pi_{+}}^{\theta} R_{\infty}^{a}$ for all $\theta$ and $\dot{\mathrm{E}}_{x}^{\theta} R_{b}^{a} \rightarrow g(\theta)$ uniformly in $\theta \in\left(0, \theta^{*}\right)$, and an elementary analysis theorem (see Apostol (1974, Theorem 9.13)) implies that $\mathrm{E}_{x}^{\theta} R_{b}^{a}$ is differentiable in $\theta \in\left(0, \theta^{*}\right)$ and $\dot{\mathrm{E}}_{\pi_{+}}^{\theta} R_{\infty}^{a}=g(\theta)$. Substituting $\dot{\mathrm{E}}_{\pi_{+}}^{\theta} R_{\infty}^{a}$ for $g(\theta)$ in (4.20) gives (4.19).

\section{Proof of Theorem 2.1}

To prove Theorem 2.1 we need to obtain the following three lemmas first. Lemma 5.1 presents a first-order approximation of (2.14) and (2.15), which extends Lemma 10.27 of Siegmund (1985) to Markov random walks. Lemma 5.2 gives the rate of convergence for the renewal measures, and Lemma 5.3 provides the rate of convergence for the distributions of overshoot on the descending ladder Markov random walk.

For given $\theta>0$ and for $x \in \mathcal{X}$, define the renewal measure $\tilde{U}_{x,-}^{\theta}$ by

$$
\tilde{U}_{x,-}^{\theta}(A, B):=\sum_{n=0}^{\infty} \tilde{\mathbf{P}}_{x}^{\theta}\left\{\tilde{\tau}_{-}^{n}<\infty, \tilde{X}_{\tilde{\tau}_{-}^{n}} \in A,-\tilde{S}_{\tilde{\tau}_{-}^{n}} \in B\right\}
$$

for all $A \in \mathcal{A}$ and Borel subsets $B \subset[0, \infty)$. We simply denote it as $\tilde{U}_{x,-}^{\theta}(B)$ if $A=\mathcal{X}$ and denote it as $\tilde{U}_{x,-}^{\theta}(v)$ if $B=[0, v)$. 
Lemma 5.1. Let $\left\{\left(X_{n}, S_{n}\right), n \geq 0\right\}$ be a Markov random walk satisfying Assumptions 2.1-2.6 and denote by $\left\{\left(X_{n}^{\theta}, S_{n}^{\theta}\right), n \geq 0\right\}$ the Markov random walk induced by (2.13) with $\mathrm{E}_{\pi}^{\theta} S_{1}>0$. Then, for any $a>0$, we have

$$
\lim _{\theta \downarrow 0} \mu_{\theta} \mathrm{E}_{\pi_{+}}^{\theta}\left(\tau_{+} S_{\tau_{+}}^{a}\right)=\frac{1}{a+1} \mathrm{E}_{\pi_{+}} S_{\tau_{+}}^{a+1}
$$

Hence, as $\theta \downarrow 0$,

$$
\mathrm{E}_{\pi_{+}}^{\theta} S_{\tau_{+}}^{a}=\mathrm{E}_{\pi_{+}} S_{\tau_{+}}^{a}+\left(\frac{a}{a+1} \mathrm{E}_{\pi_{+}} S_{\tau_{+}}^{a+1}+\mathrm{E}_{\pi_{+}}\left(S_{\tau_{+}}^{a} \alpha_{1}\left(X_{\tau_{+}}\right)\right)\right) \theta+o(\theta) .
$$

Proof. To prove (5.2) holds for $a>0$, we first assume that $\theta>0$ is fixed. Recalling $Q_{x}^{\theta}(\mathrm{d} v, \mathrm{~d} z ; b)$ defined in $(4.1)$, for $b=0$, we have

$$
Q_{x}^{\theta}(\mathrm{d} z, \mathrm{~d} v ; 0)=\sum_{n=0}^{\infty} \mathrm{P}_{x}^{\theta}\left\{\tau_{+}>n, X_{n} \in \mathrm{d} z,-S_{n} \in \mathrm{d} v\right\} .
$$

Then by (4.6), with $b=0$, we have

$$
\operatorname{cov}_{\pi_{+}}^{\theta}\left(\tau_{+}, S_{\tau_{+}}^{a}\right)=\int_{x \in \mathcal{X}} \int_{z \in \mathcal{X}} \int_{0}^{\infty}\left\{\mathrm{E}_{z}^{\theta} R_{v}^{a}-\mathrm{E}_{z}^{\theta} S_{\tau_{+}}^{a}\right\} Q_{x}^{\theta}(\mathrm{d} z, \mathrm{~d} v ; 0) \mathrm{d} \pi_{+}(x) .
$$

Applying duality to obtain $Q_{x}^{\theta}(\mathrm{d} z, \mathrm{~d} v ; 0)=\tilde{U}_{z,-}^{\theta}(\mathrm{d} x, \mathrm{~d} v)$, this implies that

$$
\begin{aligned}
\operatorname{cov}_{\pi_{+}}^{\theta}\left(\tau_{+}, S_{\tau_{+}}^{a}\right)= & \int_{x \in \mathcal{X}} \int_{z \in \mathcal{X}} \int_{0}^{\infty}\left(\mathrm{E}_{z}^{\theta} R_{v}^{a}\right) \tilde{U}_{z,-}^{\theta}(\mathrm{d} x, \mathrm{~d} v) \mathrm{d} \pi_{+}(z) \\
& -\int_{x \in \mathcal{X}} \int_{z \in \mathcal{X}} \int_{0}^{\infty}\left(\mathrm{E}_{z}^{\theta} S_{\tau_{+}}^{a}\right) Q_{x}^{\theta}(\mathrm{d} z, \mathrm{~d} v ; 0) \mathrm{d} \pi_{+}(x) \\
= & \int_{z \in \mathcal{X}} \int_{0}^{\infty}\left(\mathrm{E}_{z}^{\theta} R_{v}^{a}\right) \tilde{U}_{z,-}^{\theta}(\mathrm{d} v) \mathrm{d} \pi_{+}(z)-\left(\mathrm{E}_{\pi_{+}}^{\theta} S_{\tau_{+}}^{a}\right)\left(\mathrm{E}_{\pi_{+}}^{\theta} \tau_{+}\right),
\end{aligned}
$$

where $\tilde{U}_{z,-}^{\theta}(\mathrm{d} v):=\int_{x \in X} \tilde{U}_{z,-}^{\theta}(\mathrm{d} x, \mathrm{~d} v)$. By making use of (5.6) and the definition of $\operatorname{cov}_{\pi_{+}}^{\theta}\left(\tau_{+}, S_{\tau_{+}}^{a}\right)$, we have

$$
\begin{aligned}
\mu_{\theta} \mathrm{E}_{\pi_{+}}^{\theta}\left(\tau_{+} S_{\pi_{+}}^{a}\right) & =\mu_{\theta} \int_{z \in \mathcal{X}} \int_{0}^{\infty}\left(\mathrm{E}_{z}^{\theta} R_{v}^{a}\right) \tilde{U}_{z,-}^{\theta}(\mathrm{d} v) \mathrm{d} \pi_{+}(z) \\
& =\mu_{\theta}\left(\mathrm{E}_{\pi_{+}}^{\theta} R_{\infty}^{a}\right) \mathrm{E}_{\pi_{+}}^{\theta} \tau_{+}+\mu_{\theta} \int_{z \in \mathcal{X}} \int_{0}^{\infty}\left(\mathrm{E}_{z}^{\theta} R_{v}^{a}-\mathrm{E}_{\pi_{+}}^{\theta} R_{\infty}^{a}\right) \tilde{U}_{z,-}^{\theta}(\mathrm{d} v) \mathrm{d} \pi_{+}(z) \\
& =\frac{1}{a+1} \mathrm{E}_{\pi_{+}}^{\theta} S_{\tau_{+}}^{a+1}+O(\theta)
\end{aligned}
$$

Note that the last equality in (5.7) uses the facts that $\mathrm{E}_{\pi_{+}}^{\theta} R_{\infty}^{a}=\mathrm{E}_{\pi_{+}}^{\theta} S_{\tau_{+}}^{a+1} /(a+1) \mathrm{E}_{\pi_{+}}^{\theta} S_{\tau_{+}}$and $\mu_{\theta}=\theta+o(\theta)$, and Corollary 3.1.

Next, we prove (5.3) from

$$
\mathrm{E}_{x}^{\theta} S_{\tau_{+}}^{a}=\mathrm{E}_{x}\left\{S_{\tau_{+}}^{a} \exp \left[\theta S_{\tau_{+}}-\tau_{+} \Lambda(\theta)\right] \frac{r\left(X_{\tau_{+}} ; \theta\right)}{r(x ; \theta)}\right\}
$$


By the dominated convergence theorem it follows that $\lim _{\theta \downarrow 0} \mathrm{E}_{x}^{\theta} S_{\tau_{+}}^{a}=\mathrm{E}_{x} S_{\tau_{+}}^{a}$. Also, $f(\theta)=$ $\mathrm{E}_{x}^{\theta}\left(S_{\tau_{+}}^{a}\right)$ is continuously differentiable for small positive $\theta$ and

$$
\dot{f}(\theta)=\mathrm{E}_{x}^{\theta}\left\{S_{\tau_{+}}^{a}\left[\left(S_{\tau_{+}}-\tau_{+} \mu_{\theta}\right)+\frac{\dot{r}\left(X_{\tau_{+}} ; \theta\right) r(x ; \theta)-r\left(X_{\tau_{+}} ; \theta\right) \dot{r}(x ; \theta)}{r\left(X_{\tau_{+}} ; \theta\right) r(x ; \theta)}\right]\right\} .
$$

Since $f\left(\theta_{1}\right)=f(\theta)+\left(\theta_{1}-\theta\right) \dot{f}(\theta)+\int_{\theta}^{\theta_{1}}[\dot{f}(\alpha)-\dot{f}(\theta)] \mathrm{d} \alpha$, we obtain the result by letting $\theta \rightarrow 0$.

Lemma 5.2. Let $\left\{\left(X_{n}, S_{n}\right), n \geq 0\right\}$ be a Markov random walk satisfying Assumptions 2.1-2.6 and denote by $\left\{\left(X_{n}^{\theta}, S_{n}^{\theta}\right), n \geq 0\right\}$ the Markov random walk induced by (2.13) with $\mathrm{E}_{\pi}^{\theta} S_{1}>0$. Then, for any $A \in \mathcal{A}$ and Borel subsets $B \subset[0, \infty)$,

$$
\tilde{U}_{x,-}^{\theta}(A, B) \rightarrow \tilde{U}_{x,-}(A, B) \quad \text { as } \theta \downarrow 0,
$$

where $\tilde{U}_{x,-}^{\theta}(A, B)$ is as defined in (5.1) and $\tilde{U}_{x,-}(A, B)$ is as defined in (2.5).

Proof. For given $A \in \mathcal{A}$ and Borel subsets $B \subset[0, \infty)$. By Wald's likelihood ratio identity for the time-reversed descending ladder Markov random walk, for any $n \geq 0$, we have

$$
\begin{aligned}
\mathrm{P}_{x}^{\theta}\left\{\tilde{\tau}_{-}^{n}<\infty, \tilde{X}_{\tilde{\tau}_{-}^{n}} \in A,-\tilde{S}_{\tilde{\tau}_{-}^{n}} \in B\right\} \\
\quad=\mathrm{E}_{x}\left\{\exp \left[\theta \tilde{S}_{\tilde{\tau}_{-}^{n}}-\tilde{\tau}_{-}^{n} \tilde{\Lambda}_{-}(\theta)\right] \frac{\tilde{r}_{-}\left(\tilde{X}_{\tilde{\tau}_{-}^{n}} ; \theta\right)}{\tilde{r}_{-}(x ; \theta)} ; \tilde{X}_{\tilde{\tau}_{-}^{n}} \in A,-\tilde{S}_{\tilde{\tau}_{-}^{n}} \in B\right\}
\end{aligned}
$$

where $\tilde{\Lambda}_{-}(\theta)$ and $\tilde{r}_{-}(\underset{\tilde{S}}{x} ; \theta)$ are as defined in $(2.13)$ for the transition probability of the Markov random walk $\left\{\left(\tilde{X}_{\tilde{\tau}_{-}^{n}}, \tilde{S}_{\tilde{\tau}_{-}^{n}}\right), n \geq 0\right\}$. However, By Proposition 1 of Fuh $(2004), \tilde{r}_{-}(x ; \theta) \rightarrow 1$ as $\theta \downarrow 0$. Therefore, as $\theta \downarrow 0$,

$$
\exp \left[\theta \tilde{S}_{\tilde{\tau}_{-}^{n}}-\tilde{\tau}_{-}^{n} \tilde{\Lambda}_{-}(\theta)\right] \frac{\tilde{r}_{-}\left(\tilde{X}_{\tilde{\tau}_{-}^{n}} ; \theta\right)}{\tilde{r}_{-}(x ; \theta)} \rightarrow 1
$$

Hence, as $\theta \downarrow 0$,

$$
\mathrm{P}_{x}^{\theta}\left\{\tilde{\tau}_{-}^{n}<\infty, \tilde{X}_{\tilde{\tau}_{-}^{n}} \in A,-\tilde{S}_{\tilde{\tau}_{-}^{n}} \in B\right\} \rightarrow \mathrm{P}_{x}\left\{\tilde{X}_{\tilde{\tau}_{-}^{n}} \in A,-\tilde{S}_{\tilde{\tau}_{-}^{n}} \in B\right\} .
$$

Since $\tilde{r}_{-}(x ; \theta)=1+O(\theta)$ as $\theta \downarrow 0$, there exists a $\delta>0$ and $c>0$ such that $\left|\tilde{r}_{-}\left(\tilde{X}_{\tilde{\tau}_{-}^{n}} ; \theta\right) / \tilde{r}_{-}(x ; \theta)\right|<1+c \theta$ for $0<\theta<\delta$. Together with the fact that $\exp \left[\theta \tilde{S}_{\tilde{\tau}_{-}^{n}-}\right.$ $\left.\tilde{\tau}_{-}^{n} \tilde{\Lambda}_{-}(\theta)\right] \uparrow 1$ as $\theta \downarrow 0$, we obtain, for $0<\theta<\delta$,

$$
\mathrm{P}_{x}^{\theta}\left\{\tilde{\tau}_{-}^{n}<\infty, \tilde{X}_{\tilde{\tau}_{-}^{n}} \in A,-\tilde{S}_{\tilde{\tau}_{-}^{n}} \in B\right\} \leq(1+c \theta) \mathrm{P}_{x}\left\{\tilde{X}_{\tilde{\tau}_{-}^{n}} \in A,-\tilde{S}_{\tilde{\tau}_{-}^{n}} \in B\right\}
$$

Note that $\tilde{U}_{x,-}(A, B)<\infty$ for each $B \subset[0, \infty)$ with finite measure. Using (5.9), summing over $n \geq 0$, and applying the dominated convergence theorem, we obtain (5.8).

The following lemma is a key lemma in the proof of Theorem 2.1. The proof involves the perturbation theory, which is summarized in Fuh (2004), and a detailed analysis. 
Lemma 5.3. Let $\left\{\left(X_{n}, S_{n}\right), n \geq 0\right\}$ be a Markov random walk satisfying Assumptions 2.1-2.6 and denote by $\left\{\left(X_{n}^{\theta}, S_{n}^{\theta}\right), n \geq 0\right\}$ the Markov random walk induced by (2.13) with $\mathrm{E}_{\pi}^{\theta} S_{1}>0$. Then, for any $x \in \mathcal{X}$,

$$
\int_{[0, \infty)}\left(\mathrm{E}_{x}^{\theta} R_{b}^{a}-\mathrm{E}_{\pi_{+}}^{\theta} R_{\infty}^{a}\right) \tilde{U}_{x,-}^{\theta}(\mathrm{d} b)=\alpha_{x}^{a}+O(\theta) \text { as } \theta \downarrow 0,
$$

where $\alpha_{x}^{a}$ is as defined in (2.7).

Proof. Let $f_{x}^{\theta}(b):=\mathrm{E}_{x}^{\theta} R_{b}^{a}-\mathrm{E}_{\pi_{+}}^{\theta} R_{\infty}^{a}$ and $f_{x}(b):=\mathrm{E}_{x} R_{b}^{a}-\mathrm{E}_{\pi_{+}} R_{\infty}^{a}$, and write

$$
\begin{aligned}
\int_{[0, \infty)}\left(\mathrm{E}_{x}^{\theta} R_{b}^{a}-\mathrm{E}_{\pi_{+}}^{\theta} R_{\infty}^{a}\right) \tilde{U}_{x,-}^{\theta}(\mathrm{d} b)-\alpha_{x}^{a}= & \int_{[0, \infty)} f_{x}^{\theta}(b) \tilde{U}_{x,-}^{\theta}(\mathrm{d} b)-\int_{[0, \infty)} f_{x}(b) \tilde{U}_{x,-}(\mathrm{d} b) \\
= & \int_{[0, \infty)}\left[f_{x}^{\theta}(b)-f_{x}(b)\right] \tilde{U}_{x,-}^{\theta}(\mathrm{d} b) \\
& -\int_{[0, \infty)} f_{x}(b)\left[\tilde{U}_{x,-}(\mathrm{d} b)-\tilde{U}_{x,-}^{\theta}(\mathrm{d} b)\right] \\
:= & J_{1}-J_{2} .
\end{aligned}
$$

To complete the proof we will show that $J_{1}$ and $J_{2}$ are $O(\theta)$ as $\theta \downarrow 0$.

For $J_{1}$, we rewrite $f_{x}^{\theta}(b)-f_{x}(b)=\int_{0}^{\theta} \dot{f}_{x}^{\eta}(b) \mathrm{d} \eta$, where the dot denotes differentiation with respect to $\eta$. By Corollary 4.1 there exists a $C, r>0$, and $\theta^{*}$ such that $\left|\dot{f}_{x}^{\eta}(b)\right| \leq C \mathrm{e}^{-r b}$ for all $x \in \mathcal{X}, b \geq 0$, and all $\eta \in\left(0, \theta^{*}\right]$. Therefore, letting $\theta \in\left[0, \theta^{*}\right]$, we have, for all $b \geq 0$, $\left|f_{x}^{\theta}(b)-f_{x}(b)\right| \leq \theta C \mathrm{e}^{-r b}$. This implies that there exists a $c>0$ such that

$$
\left|J_{1}\right| \leq \theta C \int_{[0, \infty)} \mathrm{e}^{-r b} \tilde{U}_{x,-}^{\theta}(\mathrm{d} b) \leq \theta C(1+c \theta)\left(\int_{[0, \infty)} \mathrm{e}^{-r b} \tilde{U}_{x,-}(\mathrm{d} b)\right),
$$

where the second inequality follows from (5.10) and Lemma 5.2. Thus, $J_{1}=O(\theta)$.

For $J_{2}$, use Lemma 5.2 to observe that $\tilde{U}_{x,-}(\mathrm{d} b)$ and $(1+c \theta) \tilde{U}_{x,-}(\mathrm{d} b)-\tilde{U}_{x,-}^{\theta}(\mathrm{d} b)$ are nonnegative measures for each $0<\theta<\delta$. From this, and the bound from Corollary 3.1, $\left|f_{x}(b)\right| \leq C \mathrm{e}^{-r_{1} b}$ for $r_{1}>0$ and $x \in \mathcal{X}$, say, we obtain

$$
\left|J_{2}\right| \leq C \int_{[0, \infty)} \mathrm{e}^{-r b}\left[(1+c \theta) \tilde{U}_{x,-}(\mathrm{d} b)-\tilde{U}_{x,-}^{\theta}(\mathrm{d} b)\right] \quad \text { for all } 0<r<r_{1} .
$$

Now, there exists a $\theta^{*}>0$ and $r_{2}>0$ such that, for $0<r<r_{2}$, we can define $\tilde{\lambda}_{-}^{\theta}(r), \tilde{v}_{*,-}^{\theta}$, and $\tilde{\boldsymbol{Q}}_{r,-}^{\theta}$ uniformly for $\theta \in\left(0, \theta^{*}\right)$, as (2.13) for the transition probability of the Markov random walk $\left\{\left(\tilde{X}_{\tilde{\tau}_{-}^{n}}^{\theta}, \tilde{S}_{\tilde{\tau}^{n}}^{\theta}\right), n \geq 0\right\}$ under the condition of $\left\{\tilde{\tau}_{-}<\infty\right\}$. Let $v$ be an initial distribution degenerated at $\bar{x}$ and $h_{A}(r):=\mathbf{1}_{\{x \in A\}}$, then we have

$$
\begin{aligned}
& \int_{[0, \infty)} \mathrm{e}^{-r b} \tilde{U}_{x,-}^{\theta}(\mathrm{d} b)=\sum_{n=0}^{\infty} \int_{[0, \infty)} \mathrm{e}^{-r b} \mathrm{P}_{x}^{\theta}\left\{\tilde{\tau}_{-}^{n}<\infty, \tilde{X}_{\tilde{\tau}_{-}^{n}} \in \mathcal{X},-\tilde{S}_{\tilde{\tau}_{-}^{n}} \in \mathrm{d} b\right\} \\
& =\sum_{n=0}^{\infty} \mathrm{E}_{x}^{\theta}\left\{\exp \left(r \tilde{S}_{\tilde{\tau}_{-}^{n}}\right) ; \tilde{\tau}_{-}^{n}<\infty, \tilde{X}_{\tilde{\tau}_{-}^{n}} \in \mathcal{X}\right\} \\
& =\left(1-\tilde{\lambda}_{-}^{\theta}(r)\right)^{-1} \tilde{\nu}_{*,-}^{\theta} \tilde{\boldsymbol{Q}}_{r,-}^{\theta} h_{\chi}(r)+\eta^{\theta}(r),
\end{aligned}
$$


where $\eta^{\theta}(r)=\sum_{n=0}^{\infty} \tilde{v}_{*,-}^{\theta} \tilde{v}_{*,-}^{\theta}\left(\tilde{\boldsymbol{P}}_{r,-}^{\theta}\right)^{n}\left(I-\tilde{\boldsymbol{Q}}_{r,-}^{\theta}\right) h_{\mathcal{X}}(r)$. Note that the last equality follows from Proposition 1 of Fuh (2004).

Choosing $r^{*}=\min \left\{r_{1}, r_{2}\right\}$ and considering $r \in\left(0, r^{*}\right),(5.11)$ implies that

$$
\begin{aligned}
\int_{[0, \infty)} & \mathrm{e}^{-r b}\left[(1+c \theta) \tilde{U}_{x,-}(\mathrm{d} b)-\tilde{U}_{x,-}^{\theta}(\mathrm{d} b)\right] \\
= & \frac{\tilde{\lambda}_{-}(r)-\tilde{\lambda}_{-}^{\theta}(r)}{\left[1-\tilde{\lambda}_{-}(r)\right]\left[1-\tilde{\lambda}_{-}^{\theta}(r)\right]} \tilde{\boldsymbol{v}}_{*,-} \tilde{\boldsymbol{Q}}_{r,-} h_{X}(r)+\frac{c \theta}{1-\tilde{\lambda}_{-}(r)} \tilde{\boldsymbol{v}}_{*,-} \tilde{\boldsymbol{Q}}_{r,-} h_{X}(r) \\
& +\frac{1}{1-\tilde{\lambda}_{-}^{\theta}(r)}\left(\tilde{v}_{*,-} \tilde{\boldsymbol{Q}}_{r,-} h_{X}(r)-\tilde{v}_{*,-}^{\theta} \tilde{\boldsymbol{Q}}_{r,-}^{\theta} h_{X}(r)\right)+(1+c \theta) \eta(r)-\eta^{\theta}(r) .
\end{aligned}
$$

By Proposition 1 of Fuh (2004), $\tilde{\lambda}_{-}(r), \tilde{\lambda}_{-}^{\theta}(r), \tilde{\nu}_{*,-} \tilde{\boldsymbol{Q}}_{r,-} h_{\chi}(r), \tilde{\nu}_{*,-}^{\theta} \tilde{\boldsymbol{Q}}_{r,-}^{\theta} h_{\chi}(r), \eta(r)$, and $\eta^{\theta}(r)$ (uniformly for $\theta \in\left[0, \theta^{*}\right]$ ) have continuous derivatives for $|r|$ near 0 and

$$
\tilde{\lambda}_{-}(r)=1+\left(\tilde{\mathrm{E}}_{\pi_{-}} \tilde{S}_{\tilde{\tau}_{-}}\right) r+O\left(r^{2}\right), \quad \tilde{\lambda}_{-}^{\theta}(r)=1+\left(\tilde{\mathrm{E}}_{\pi_{-}}^{\theta} \tilde{S}_{\tilde{\tau}_{-}}\right) r+O\left(r^{2}\right) .
$$

Moreover, we have, as $\theta \rightarrow 0$,

$$
\tilde{\boldsymbol{v}}_{*,-}^{\theta} \tilde{\boldsymbol{Q}}_{r,-}^{\theta} h_{\chi}(r) \rightarrow \tilde{\boldsymbol{v}}_{*,-} \tilde{\boldsymbol{Q}}_{r,-} h_{\chi}(r) \quad \text { and } \quad \eta^{\theta}(r) \rightarrow \eta(r) .
$$

Now, for fixed $r \in\left(0, r^{*}\right),(5.13)$ and (5.14) imply that, as $\theta \rightarrow 0,(5.12)$ equals

$$
\begin{aligned}
& \frac{\left(\tilde{\mathrm{E}}_{\pi_{-}} \tilde{S}_{\tilde{\tau}_{-}}-\tilde{\mathrm{E}}_{\pi_{-}}^{\theta} \tilde{S}_{\tilde{\tau}_{-}}\right) r}{\left(\tilde{\mathrm{E}}_{\pi_{-}} \tilde{S}_{\tilde{\tau}_{-}} \tilde{\mathrm{E}}_{\pi_{-}}^{\theta} \tilde{S}_{\tilde{\tau}_{-}}\right) r^{2}} \tilde{\nu}_{*,-} \tilde{\boldsymbol{Q}}_{r,-} h_{X}(r)+\frac{c \theta}{-\left(\tilde{\mathrm{E}}_{\pi_{-}} \tilde{S}_{\tilde{\tau}_{-}}\right) r+O\left(r^{2}\right)} \tilde{\mathcal{V}}_{*,-} \tilde{\boldsymbol{Q}}_{r,-} h_{X}(r) \\
& \quad+\frac{1}{\left(\tilde{\mathrm{E}}_{\pi_{-}}^{\theta} \tilde{S}_{\tilde{\tau}_{-}}\right) r+O\left(r^{2}\right)} o(\theta)+c \theta \eta(r)+o(\theta) .
\end{aligned}
$$

By making use of the same argument given above for (5.3) in Lemma 5.1, we have $\tilde{\mathrm{E}}_{\pi_{-}}^{\theta} \tilde{S}_{\tilde{\tau}_{-}}=$ $\tilde{\mathrm{E}}_{\pi_{-}} \tilde{S}_{\tilde{\tau}_{-}}+O(\theta)$. Therefore, (5.15) and (5.12) imply that $J_{2}=O(\theta)$. This completes the proof.

Proof of Theorem 2.1. First, we show that (2.14) implies that (2.15) holds. Let $a>0$ and denote $h_{x}(\theta)=\mathrm{E}_{x}^{\theta} S_{\tau_{+}}^{a}$. From

$$
\mathrm{E}_{x}^{\theta} S_{\tau_{+}}^{a}=\mathrm{E}_{x}\left\{S_{\tau_{+}}^{a} \exp \left[\theta S_{\tau_{+}}-\tau_{+} \Lambda(\theta)\right] \frac{r\left(X_{\tau_{+}} ; \theta\right)}{r(x ; \theta)}\right\},
$$

by the dominated convergence theorem it follows that $\lim _{\theta \downarrow 0} \mathrm{E}_{x}^{\theta} S_{\tau_{+}}^{a}=\mathrm{E}_{x} S_{\tau_{+}}^{a}$. Since, for any $x \in \mathcal{X}, h_{x}(\theta)$ is continuously differentiable for small positive $\theta, r(x ; \theta)=1+O(\theta)$ and $\dot{r}(x ; \theta)=\dot{r}(x ; 0)+O(\theta)$, we have

$$
\begin{aligned}
\dot{h}_{x}(\theta) & =\mathrm{E}_{x}^{\theta}\left\{S_{\tau_{+}}^{a}\left[\left(S_{\tau_{+}}-\tau_{+} \mu_{\theta}\right)+\frac{\dot{r}\left(X_{\tau_{+}} ; \theta\right) r(x ; \theta)-r\left(X_{\tau_{+}} ; \theta\right) \dot{r}(x ; \theta)}{r\left(X_{\tau_{+}} ; \theta\right) r(x ; \theta)}\right]\right\} \\
& =\mathrm{E}_{x}^{\theta}\left\{S_{\tau_{+}}^{a}\left[\left(S_{\tau_{+}}-\tau_{+} \mu_{\theta}\right)+\dot{r}\left(X_{\tau_{+}} ; 0\right)-\dot{r}(x ; 0)\right]\right\}+O(\theta) .
\end{aligned}
$$

Then, we integrate $x \in \mathcal{X}$ with respect to $\pi_{+}$in (5.16) to obtain

$$
\dot{h}_{\pi_{+}}(\theta)=\mathrm{E}_{\pi_{+}}^{\theta} S_{\tau_{+}}^{a+1}-\mu_{\theta} \mathrm{E}_{\pi_{+}}^{\theta}\left(\tau_{+} S_{\tau_{+}}^{a}\right)+\mathrm{E}_{\pi_{+}}^{\theta}\left(S_{\tau_{+}}^{a}\left(\dot{r}\left(X_{\tau_{+}} ; 0\right)-\dot{r}\left(X_{0} ; 0\right)\right)\right)+O(\theta)
$$


Since

$$
h_{x}(\theta)=h_{x}\left(\theta^{*}\right)+\left(\theta-\theta^{*}\right) \dot{h}_{x}\left(\theta^{*}\right)+\int_{\theta^{*}}^{\theta}\left[\dot{h}_{x}(\alpha)-\dot{h}_{x}\left(\theta^{*}\right)\right] \mathrm{d} \alpha,
$$

we obtain, via (5.3) in Lemma 5.1, by letting $\theta^{*} \rightarrow 0$,

$$
\mathrm{E}_{x}^{\theta} S_{\tau_{+}}^{a}=\mathrm{E}_{x} S_{\tau_{+}}^{a}+\left(\frac{a}{a+1} \mathrm{E}_{x} S_{\tau_{+}}^{a+1}+\mathrm{E}_{x}\left[S_{\tau_{+}}^{a}\left(\dot{r}\left(X_{\tau_{+}} ; 0\right)-\dot{r}(x ; 0)\right)\right]\right) \theta+o(\theta) .
$$

Then, we integrate $x \in \mathcal{X}$ with respect to $\pi_{+}$in (5.18) to obtain

$$
\mathrm{E}_{\pi_{+}}^{\theta} S_{\tau_{+}}^{a}=\mathrm{E}_{\pi_{+}} S_{\tau_{+}}^{a}+\left(\frac{a}{a+1} \mathrm{E}_{\pi_{+}} S_{\tau_{+}}^{a+1}+\mathrm{E}_{\pi_{+}}\left[S_{\tau_{+}}^{a}\left(\dot{r}\left(X_{\tau_{+}} ; 0\right)-\dot{r}(x ; 0)\right)\right]\right) \theta+o(\theta) .
$$

To obtain a further expansion of $\dot{h}_{\pi_{+}}(\theta)$, we first expand $r(x ; \theta)=1+\dot{r}(x ; \theta) \theta+O\left(\theta^{2}\right)$ and $\dot{r}(x ; \theta)=\dot{r}(x ; 0)+\ddot{r}(x ; 0) \theta+O\left(\theta^{2}\right)$, and then use $1 /(1-\theta)=1+\theta+O\left(\theta^{2}\right)$ to obtain

$$
\begin{aligned}
\frac{\dot{r}\left(X_{\tau_{+}} ; \theta\right) r(x ; \theta)-r\left(X_{\tau_{+}} ; \theta\right) \dot{r}(x ; \theta)}{r\left(X_{\tau_{+}} ; \theta\right) r(x ; \theta)}= & \left(\dot{r}\left(X_{\tau_{+}} ; 0\right)-\dot{r}(x ; 0)\right) \\
& +\left(\left(\ddot{r}\left(X_{\tau_{+}} ; 0\right)-\dot{r}^{2}\left(X_{\tau_{+}} ; 0\right)\right)\right. \\
& \left.-\left(\ddot{r}(x ; 0)-\dot{r}^{2}(x ; 0)\right)\right) \theta+O\left(\theta^{2}\right) .
\end{aligned}
$$

Denote

$$
\begin{aligned}
& \beta_{1}\left(X_{\tau_{+}}\right)=\dot{r}\left(X_{\tau_{+}} ; 0\right)-\dot{r}\left(X_{0} ; 0\right), \\
& \beta_{2}\left(X_{\tau_{+}}\right)=\left(\ddot{r}\left(X_{\tau_{+}} ; 0\right)-\dot{r}^{2}\left(X_{\tau_{+}} ; 0\right)\right)-\left(\ddot{r}\left(X_{0} ; 0\right)-\dot{r}^{2}\left(X_{0} ; 0\right)\right) .
\end{aligned}
$$

By making use of (2.14), (5.19), and (5.20), with $a$ replaced by $a+1$, we have

$$
\begin{aligned}
\dot{h}_{\pi_{+}}(\theta)= & \mathrm{E}_{\pi_{+}} S_{\tau_{+}}^{a+1}+\left(\frac{a+1}{a+2} \mathrm{E}_{\pi_{+}} S_{\tau_{+}}^{a+2}+\mathrm{E}_{\pi_{+}}\left[S_{\tau_{+}}^{a+1}\left(\dot{r}\left(X_{\tau_{+}} ; 0\right)-\dot{r}\left(X_{0} ; 0\right)\right)\right]\right) \theta+o(\theta) \\
& -\frac{1}{a+1} \mathrm{E}_{\pi_{+}} S_{\tau_{+}}^{a+1}-\left(\frac{1}{a+2} \mathrm{E}_{\pi_{+}} S_{\tau_{+}}^{a+2}+\alpha^{a}\right)+O\left(\theta^{2}\right) \\
& +\mathrm{E}_{\pi_{+}}\left(S_{\tau_{+}}^{a}\left(\dot{r}\left(X_{\tau_{+}} ; 0\right)-\dot{r}\left(X_{0} ; 0\right)\right)\right) \\
& +\mathrm{E}_{\pi_{+}}\left(S_{\tau_{+}}^{a}\left[\left(\ddot{r}\left(X_{\tau_{+}} ; 0\right)-\ddot{r}\left(X_{0} ; 0\right)\right)-\left(\dot{r}^{2}\left(X_{\tau_{+}} ; 0\right)-\dot{r}^{2}\left(X_{0} ; 0\right)\right)\right]\right) \theta+O\left(\theta^{2}\right) \\
= & \frac{a}{a+1} \mathrm{E}_{\pi_{+}} S_{\tau_{+}}^{a+1}+\mathrm{E}_{\pi_{+}}\left(S_{\tau_{+}}^{a} \beta_{1}\left(X_{\tau_{+}}\right)\right)+\left(\frac{a}{a+2} \mathrm{E}_{\pi_{+}} S_{\tau_{+}}^{a+2}-\alpha^{a}\right) \theta \\
& +\mathrm{E}_{\pi_{+}}\left(S_{\tau_{+}}^{a+1} \beta_{1}\left(X_{\tau_{+}}\right)+S_{\tau_{+}}^{a} \beta_{2}\left(X_{\tau_{+}}\right)\right) \theta+o(\theta) .
\end{aligned}
$$

Since, for some $\varepsilon>0, h_{\pi_{+}}$is continuously differentiable on $(0, \varepsilon)$ and continuous on $[0, \varepsilon]$, for small $\theta$, we have

$$
h_{\pi_{+}}(\theta)=h_{\pi_{+}}(0)+\int_{0}^{\theta} \dot{h}_{\pi_{+}}\left(\theta^{\prime}\right) \mathrm{d} \theta^{\prime}
$$


Then, replacing $\dot{h}_{\pi_{+}}\left(\theta^{\prime}\right)$ in (5.22) by that in (5.21), a simple calculation yields

$$
\begin{aligned}
\mathrm{E}_{\pi_{+}}^{\theta} S_{\tau_{+}}^{a}= & h_{\pi_{+}}(\theta) \\
= & \mathrm{E}_{\pi_{+}} S_{\tau_{+}}^{a}+\left(\frac{a}{a+1}\left(\mathrm{E}_{\pi_{+}} S_{\tau_{+}}^{a+1}\right)+\mathrm{E}_{\pi_{+}}\left(S_{\tau_{+}}^{a} \beta_{1}\left(X_{\tau_{+}}\right)\right)\right) \theta \\
& +\frac{1}{2}\left(\frac{a}{a+2} \mathrm{E}_{\pi_{+}} S_{\tau_{+}}^{a+2}-\alpha^{a}\right) \theta^{2} \\
& +\frac{1}{2} \mathrm{E}_{\pi_{+}}\left(S_{\tau_{+}}^{a+1} \beta_{1}\left(X_{\tau_{+}}\right)+S_{\tau_{+}}^{a} \beta_{2}\left(X_{\tau_{+}}\right)\right) \theta^{2}+o\left(\theta^{2}\right) \\
= & \mathrm{E}_{\pi_{+}} S_{\tau_{+}}^{a}+\left(\frac{a}{a+1}\left(\mathrm{E}_{\pi_{+}} S_{\tau_{+}}^{a+1}\right)+\mathrm{E}_{\pi_{+}}\left(S_{\tau_{+}}^{a} \beta_{1}\left(X_{\tau_{+}}\right)\right)\right) \theta+O\left(\theta^{2}\right) .
\end{aligned}
$$

Now changing $a$ to $a+1$ in (5.23) and recalculating (5.21), we have

$$
\begin{aligned}
\dot{h}_{\pi_{+}}(\theta)= & \frac{a}{a+1} \mathrm{E}_{\pi_{+}} S_{\tau_{+}}^{a+1}+\mathrm{E}_{\pi_{+}}\left(S_{\tau_{+}}^{a} \beta_{1}\left(X_{\tau_{+}}\right)\right)+\left(\frac{a}{a+2} \mathrm{E}_{\pi_{+}} S_{\tau_{+}}^{a+2}-\alpha^{a}\right) \theta \\
& +\mathrm{E}_{\pi_{+}}\left(S_{\tau_{+}}^{a+1} \beta_{1}\left(X_{\tau_{+}}\right)+S_{\tau_{+}}^{a} \beta_{2}\left(X_{\tau_{+}}\right)\right) \theta+O\left(\theta^{2}\right) .
\end{aligned}
$$

Substituting (5.24) into (5.22), we obtain (2.15).

Next, we will prove that (2.14) holds. First we assume that $\theta>0$ is fixed. Using the same argument as given above for (5.4)-(5.7), we obtain

$$
\begin{aligned}
\mu_{\theta} \mathrm{E}_{\pi_{+}}^{\theta}\left(\tau_{+} S_{\tau_{+}}^{a}\right) & =\mu_{\theta} \int_{z \in \mathcal{X}} \int_{0}^{\infty}\left(\mathrm{E}_{z}^{\theta} R_{v}^{a}\right) \tilde{U}_{z,-}^{\theta}(\mathrm{d} v) \mathrm{d} \pi_{+}(z) \\
& =\mu_{\theta}\left(\mathrm{E}_{\pi_{+}}^{\theta} R_{\infty}^{a}\right) \mathrm{E}_{\pi_{+}}^{\theta} \tau_{+}+\mu_{\theta} \int_{z \in \mathcal{X}} \int_{0}^{\infty}\left(\mathrm{E}_{z}^{\theta} R_{v}^{a}-\mathrm{E}_{\pi_{+}}^{\theta} R_{\infty}^{a}\right) \tilde{U}_{z,-}^{\theta}(\mathrm{d} v) \mathrm{d} \pi_{+}(z) \\
& =\frac{1}{a+1} \mathrm{E}_{\pi_{+}}^{\theta} S_{\tau_{+}}^{a+1}+\alpha^{a} \theta+O\left(\theta^{2}\right)
\end{aligned}
$$

Note that the last equality in (5.25) uses the facts that $\mathrm{E}_{\pi_{+}}^{\theta} R_{\infty}^{a}=\mathrm{E}_{\pi_{+}}^{\theta} S_{\tau_{+}}^{a+1} /(a+1) \mathrm{E}_{\pi_{+}}^{\theta} S_{\tau_{+}}$ and $\mu=\theta+O\left(\theta^{2}\right)$, and Lemma 5.3. Now, we need to establish an expansion for $\mathrm{E}_{\pi_{+}}^{\theta} S_{\tau_{+}}^{a+1}$ up to $O\left(\theta^{2}\right)$. Observe that

$$
\begin{aligned}
\dot{\mathrm{E}}_{\pi_{+}}^{\theta}\left(S_{\tau_{+}}^{a+1}\right) & =\mathrm{E}_{\pi_{+}}^{\theta} S_{\tau_{+}}^{a+2}-\mu_{\theta} \mathrm{E}_{\pi_{+}}^{\theta}\left(\tau_{+} S_{\tau_{+}}^{a+1}\right)+\mathrm{E}_{\pi_{+}}^{\theta}\left(S_{\tau_{+}}^{a+1}\left(\dot{r}\left(X_{\tau_{+}} ; 0\right)-\dot{r}(x ; 0)\right)\right) \\
& =\mathrm{E}_{\pi_{+}}^{\theta} S_{\tau_{+}}^{a+2}-\frac{1}{a+2} \mathrm{E}_{\pi_{+}}^{\theta} S_{\tau_{+}}^{a+2}+\mathrm{E}_{\pi_{+}}^{\theta}\left(S_{\tau_{+}}^{a+1}\left(\dot{r}\left(X_{\tau_{+}} ; 0\right)-\dot{r}(x ; 0)\right)\right)+O(\theta) \\
& =\frac{a+1}{a+2} \mathrm{E}_{\pi_{+}}^{0} S_{\tau_{+}}^{a+2}+\mathrm{E}_{\pi_{+}}\left(S_{\tau_{+}}^{a+1} \beta_{1}\left(X_{\tau_{+}}\right)\right)+O(\theta),
\end{aligned}
$$

where the first equality is from (5.17), and the second and third equalities are from (5.7) and Lemma 5.1, respectively. Integrating the last display of (5.26) gives

$$
\mathrm{E}_{\pi_{+}}^{\theta}\left(S_{\tau_{+}}^{a+1}\right)=\mathrm{E}_{\pi_{+}}\left(S_{\tau_{+}}^{a+1}\right)+\left(\frac{a+1}{a+2} \mathrm{E}_{\pi_{+}} S_{\tau_{+}}^{a+2}+\mathrm{E}_{\pi_{+}}\left(S_{\tau_{+}}^{a+1} \beta_{1}\left(X_{\tau_{+}}\right)\right)\right) \theta+O\left(\theta^{2}\right) .
$$

Substituting (5.27) into (5.7) yields (2.14). 
To complete the proof, it remains to show that (2.14) and (2.15) still hold when $\beta_{1}\left(X_{\tau_{+}}\right)$and $\beta_{2}\left(X_{\tau_{+}}\right)$are replaced by $\alpha_{1}\left(X_{\tau_{+}}\right)$and $\alpha_{2}\left(X_{\tau_{+}}\right)$defined in (2.10) and (2.11), respectively. This will be shown in the next lemma.

Recall the definitions of $g_{1}$ and $g_{2}$ given in (2.8) and (2.9), respectively.

Lemma 5.4. Assume that the conditions of Theorem 2.1 hold. Then, $\dot{r}(x ; 0)$ and $\ddot{r}(x ; 0)$ are bounded on $\mathcal{X}$ and, for any $x \in \mathcal{X}$, there exist constants $c_{1}$ and $c_{2}$ such that

$$
\begin{gathered}
\dot{r}(x ; 0)=g_{1}(x)+c_{1}, \\
\ddot{r}(x ; 0)-\dot{r}^{2}(x ; 0)=g_{2}(x)+c_{2} .
\end{gathered}
$$

Proof. To establish (5.28) and (5.29), we simply assume that the random variable $\xi_{1}$ takes positive values, since the extension to the general case is straightforward via ladder random variables. Since $r(\cdot ; \theta)$ is an eigenfunction of $\lambda(\theta)$ with respect to the operator $\boldsymbol{P}_{\theta}$, we have $\boldsymbol{P}_{\theta} r(x ; \theta)=\lambda(\theta) r(x ; \theta)$, which implies that $\mathrm{E}_{x}\left\{\mathrm{e}^{\theta \xi_{1}} r(x ; \theta)\right\}=\lambda(\theta) r(x ; \theta)$. By Proposition 1 of Fuh (2004), there exists a $\delta>0$ such that both $\lambda(\theta)$ and $r(\cdot ; \theta)$ are analytic functions for $|\theta|<\delta$. Note that $\mu=\dot{\lambda}(0)>0$ under the assumption that $\xi_{1}$ takes positive values. A one-term Taylor expansion for $\lambda(\theta)$ and $r(x ; \theta)$ with respect to $\theta$ around 0 entails $\lambda(\theta)=1+\mu \theta+O\left(\theta^{2}\right)$ and $r(x ; \theta)=1+\dot{r}(x ; 0) \theta+O\left(\theta^{2}\right)$. Therefore, for any $x \in \mathcal{X}$,

$\mathrm{E}_{x}\left(1+\xi_{1} \theta+O\left(\theta^{2}\right)\right)\left(1+\dot{r}\left(X_{1} ; 0\right) \theta+O\left(\theta^{2}\right)\right)=\left(1+\mu \theta+O\left(\theta^{2}\right)\right)\left(1+\dot{r}(x ; 0) \theta+O\left(\theta^{2}\right)\right)$.

Matching the coefficient of $\theta$ in (5.30), we obtain

$$
\dot{r}(x ; 0)-\mathrm{E}_{x} \dot{r}\left(X_{1} ; 0\right)=\mathrm{E}_{x} \xi_{1}-\mu .
$$

By Assumptions 2.1-2.4 and $\mathrm{E}_{\pi}\left|\mathrm{E}_{x} \xi_{1}-\mu\right|<\infty$, the existence and boundedness of the solution $\dot{r}(x ; 0)$ for the Poisson equation (5.30) follows from (17.38) and Theorem 17.4.2 of Meyn and Tweedie (1993). Furthermore, $|\dot{r}(x ; 0)| \leq\left|\mathrm{E}_{x} \xi_{1}-\mu\right|$. Hence, (5.28) follows from (5.31).

To prove (5.29), note that $\mu=\dot{\Lambda}(0)>0$ and $\sigma^{2}=\ddot{\lambda}(0)-\mu^{2}$. A two-term Taylor expansion for $\lambda(\theta)$ and $r(x ; \theta)$ with respect to $\theta$ around 0 entails $\lambda(\theta)=1+\mu \theta+\ddot{\lambda}(0) \theta^{2} / 2+O\left(\theta^{3}\right)$ and $r(x ; \theta)=1+\dot{r}(x ; 0) \theta+\ddot{r}(x ; 0) \theta^{2} / 2+O\left(\theta^{3}\right)$. Therefore,

$$
\begin{aligned}
\mathrm{E}_{x}(1 & \left.+\xi_{1} \theta+\frac{\xi_{1}^{2} \theta^{2}}{2}+O\left(\theta^{3}\right)\right)\left(1+\dot{r}\left(X_{1} ; 0\right) \theta+\frac{\ddot{r}\left(X_{1} ; 0\right) \theta^{2}}{2}+O\left(\theta^{3}\right)\right) \\
= & \left(1+\mu \theta+\frac{\ddot{\lambda}(0) \theta^{2}}{2}+O\left(\theta^{3}\right)\right)\left(1+\dot{r}(x ; 0) \theta+\frac{\ddot{r}(x ; 0) \theta^{2}}{2}+O\left(\theta^{3}\right)\right) .
\end{aligned}
$$

Matching the coefficient of $\theta^{2}$ in (5.32), we obtain

$$
2 \mathrm{E}_{x}\left(\xi_{1} \dot{r}\left(X_{1} ; 0\right)\right)+\mathrm{E}_{x} \ddot{r}\left(X_{1} ; 0\right)+\mathrm{E}_{x} \xi_{1}^{2}=\ddot{r}(x ; 0)+\mu \dot{r}(x ; 0)+\sigma^{2}+\mu^{2} .
$$

A simple but tedious calculation via (5.33) yields

$$
\begin{aligned}
& \left(\ddot{r}(x ; 0)-\dot{r}^{2}(x ; 0)\right)-\mathrm{E}_{x}\left(\ddot{r}\left(X_{1} ; 0\right)-\dot{r}^{2}\left(X_{1} ; 0\right)\right) \\
& \quad=\mathrm{E}_{x}\left(\xi_{1}-\mu+\dot{r}\left(X_{1} ; 0\right)-\dot{r}(x ; 0)\right)^{2}-\mathrm{E}_{\pi}\left(\xi_{1}-\mu+\dot{r}\left(X_{1} ; 0\right)-\dot{r}\left(X_{0} ; 0\right)\right)^{2} .
\end{aligned}
$$


By Assumptions 2.1-2.4 and because there exists a positive constant $c$ such that $\mathrm{E}_{\pi}\left(\mathrm{E}_{x} \xi_{1}-\right.$ $\left.\mu+\mathrm{E}_{\pi} \dot{r}\left(X_{1} ; 0\right)-\dot{r}\left(X_{0} ; 0\right)\right)^{2} \leq c \sup _{x} \mathrm{E}_{x} \xi_{1}^{2}<\infty$, the existence and boundedness of the solution $\ddot{r}(x ; 0)-\dot{r}^{2}(x ; 0)$ of (5.34) follows from (17.38) and Theorem 17.4.2 of Meyn and Tweedie (1993). Furthermore, we have

$$
|\ddot{r}(x ; 0)| \leq\left(\mathrm{E}_{x} \xi_{1}-\mu+\mathrm{E}_{\pi} \dot{r}\left(X_{1} ; 0\right)-\dot{r}(x ; 0)\right)^{2} .
$$

\section{Acknowledgements}

The author thanks an Associate Editor and an anonymous referee for their valuable comments. This research was partially supported by NSC 95-2118-M-001-008.

\section{References}

Alsmeyer, G. (1997). The Markov renewal theorem and related results. Markov Proc. Relat. Fields 3, 103-127. Alsmeyer, G. (2000). The ladder variables of a Markov random walk. Theoret. Prob. Math. Statist. 20, $151-168$. Apostol, T. M. (1974). Mathematical Analysis. Addison-Wesley, Reading, MA.

ARNDT, K. (1980). Asymptotic properties of the distribution of the supremum of a random walk on a Markov chain. Theory Prob. Appl. 25, 309-323.

Asmussen, S. (1989a). Aspects of matrix Wiener-Hopf factorization in applied probability. Math. Scientist. 14, 101-116.

Asmussen, S. (1989b). Risk theory in a Markov environment. Scand. Actuarial J. 1989, 69-100.

Asmussen, S. (2000). Ruin Probabilities. World Scientific, Singapore.

Asmussen, S. (2003). Applied Probability and Queues. Springer, New York.

Basseville, M. And Nikiforov, I. V. (1993). Detection of Abrupt Changes: Theory and Application. Prentice Hall, Englewood Cliffs, NJ.

Blanchet, J. and Glynn, P. (2006). Complete corrected diffusion approximations for the maximum of a random walk. Ann. Appl. Prob. 16, 951-983.

Burman, D. Y. AND SMith, D. R. (1986). An asymptotic analysis of a queueing system with Markov-modulated arrivals. Operat. Res. 34, 105-119.

Chan, H. P. And LaI, T. L. (2003). Saddlepoint approximations and nonlinear boundary crossing probabilities of Markov random walks. Ann. Appl. Prob. 13, 395-429.

Chang, J. T. (1992). On moments of the first ladder height of random walks with small drift. Ann. Appl. Prob. 2, 714-738.

Chang, J. T. And Peres, Y. (1997). Ladder heights, Gaussian random walks and the Riemann zeta function. Ann. Prob. 25, 787-802.

FuH, C. D. (1997). Corrected diffusion approximations for ruin probabilities in a Markov random walk. Adv. Appl. Prob. 29, 695-712.

FuH, C. D. (2003). SPRT and CUSUM in hidden Markov models. Ann. Statist. 31, 942-977.

FuH, C. D. (2004). Uniform Markov renewal theory and ruin probabilities in Markov random walks. Ann. Appl. Prob. 14, 1202-1241.

FuH, C. D. AND LAI, T. L. (1998). Wald's equations, first passage times and moments of ladder variables in Markov random walks. J. Appl. Prob. 35, 566-580.

FuH, C. D. And LAI, T. L. (2001). Asymptotic expansions in multidimensional Markov renewal theory and first passage times for Markov random walks. Adv. Appl. Prob. 33, 652-673.

Fuh, C. D. And Zhang, C. H. (2000). Poisson equation, moment inequalities and quick convergence for Markov random walks. Stoch. Process. Appl. 87, 53-67.

Glasserman, P. and Kou, S. (1995). Limits of first passage times to rare sets in regenerative processes. Ann. Appl. Prob. 5, 424-445.

Hoglund, T. (1991). The ruin problem for finite Markov chains. Ann. Prob. 19, 1298-1310.

Kartashov, N. V. (1996). Strong Stable Markov Chains. VSP, Utrecht.

Kesten, H. (1974). Renewal theory for functionals of a Markov chain with general state space. Ann. Prob. 2, 355-386.

Klüppelberg, C. and Pergamenshchikov, S. M. (2003). Renewal theory for functionals of a Markov chain with compact state space. Ann. Prob. 31, 2270-2300.

Lalley, S. P. (1984). Limit theorems for first-passage times in linear and non-linear renewal theory. Adv. Appl. Prob. 16, 766-803.

LoRden, G. (1970). On excess over the boundary. Ann. Math. Statist. 41, 520-527.

Lotov, V. I. (1996). On some boundary crossing problems for Gaussian random walks. Ann. Prob. 24, $2154-2171$. 
Meyn, S. P. AND Tweedie, R. L. (1993). Markov Chains and Stochastic Stability. Springer, New York.

Miller, H. D. (1962a). A matrix factorization problem in the theory of random variables defined on a finite Markov chain. Proc. Camb. Philios. Soc. 58, 268-285.

Miller, H. D. (1962b). Absorption probabilities for sums of random variables defined on a finite Markov chain. Proc. Camb. Philios. Soc. 58, 286-298.

Ney, P. And Nummelin, E. (1987). Markov additive processes. I. Eigenvalue properties and limit theorems. Ann. Prob. 15, 561-592.

Siegmund, D. (1979). Corrected diffusion approximations in certain random walk problems. Adv. Appl. Prob. 11, 701-719.

Siegmund, D. (1982). Large deviations for boundary crossing probabilities. Ann. Prob. 10, 581-588.

Siegmund, D. (1985). Sequential Analysis. Springer, New York.

Siegmund, D. (1988). Approximate tail probabilities for the maxima of some random fields. Ann. Prob. 16, $487-501$. 